\title{
ACOUSTIC TURBIDITY AS ONLINE MONITORING TOOL FOR RIVERS AND SEWER NETWORKS
}

\author{
Anne Pallarès, Philippe Schmitt, Stéphane Fischer
}

Contact:

Anne Pallarès

Université de Strasbourg - Icube, Département de Mécanique

2 rue Boussingault

67000 Strasbourg, FRANCE

anne.pallares@unistra.fr 


\section{INTRODUCTION}

The demand for better characterization of water quality in its natural environment, like rivers, or in sewer systems, has increased with the evolution of the water policy laws. An online or at least regular survey of the water networks is needed. Thus, the implementation of network survey devices is a growing practice. To monitor the pollutant load, an essential parameter is the suspended solids concentration.

Erosion, transport, and deposition of sediments is a primary and growing environmental, engineering, and agricultural issue around the world. Environmental impacts of sedimentation are various, going from mechanical obstruction to contamination by pollutants attached to and transported by sediments.

Currently, the suspended sediment concentration in water is either measured through sampling and laboratory measurement, or optical methods (nephelometric and optical backscatter sensors) (Gray and al. 2009). Also, due to the importance of flow velocities in the pollutant load behaviour, monitored water ways are often equipped with Acoustic Doppler Velocimeters (ADV) or Acoustic Doppler Current Profilers (ADCP). In addition to velocities, these devices also monitor the acoustic backscattered signal amplitude, or acoustic turbidity, which is proportional to the Suspended Sediment Concentration (SSC).

The aim of this paper is to recall the fundamental knowledge about the acoustic signal necessary for the good handling of ADVs or ADCPs. A second goal is to show that these devices can already bring valuable information without complicated data treatment.

\section{STATE OF THE ART}

Accessing the suspended sediment concentration is of interest in many fields of science. In sediment transport studies, these measurements usually rely on various types of Optical Backscatter Sensors (OBS). As shown in a review paper (Downing 2006), after adequate calibration, OBS sensors might provide an inexpensive mean to monitor the suspended solids when their size distribution and range do not change during the measurement period. One of their major drawbacks is their sensitivity to particle-size and the need to keep the sensor's windows clean. Acoustic systems are at least less care demanding.

In the field of oceanographic research, various studies on the comparison between optical and acoustic surrogates were performed as for example the two following references (Hawley 2004, Chanson et al. 2008). The acoustic measurements were usually done with ADVs or ADCPs. The results demonstrate that an acoustic system seems even more sensitive to the particle nature and size. Thus, the calibration of an acoustic Doppler system must be performed with the waters and soil materials of the natural system. Importantly, the calibration of the acoustic Doppler system is specific to the instrument itself.

However, in the field of urban drainage monitoring, with nearly no calibration of the acoustic system, but with a crude knowledge of the acoustical characteristics of particles, some trials of the use of acoustic systems were performed (Pallarès et al. 2011). As shown figure 1, a good agreement between sampling, optical turbidity measurement and acoustic total suspended sediment concentration measurement is shown. 


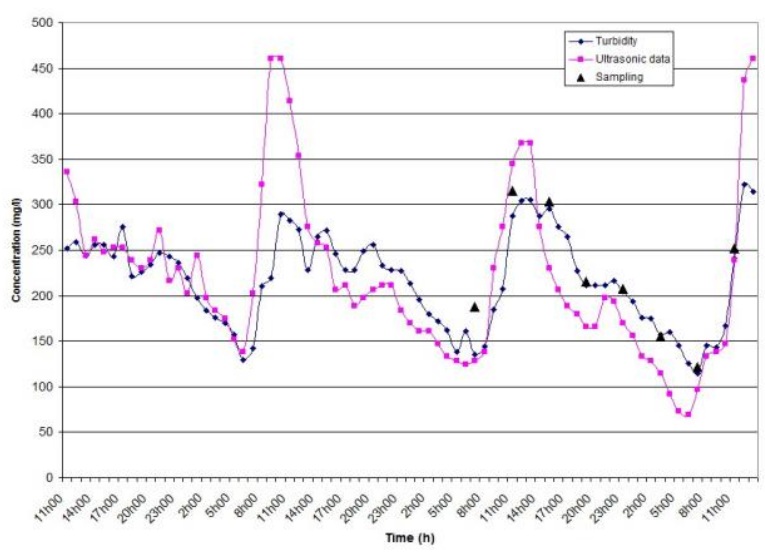

Figure 1 Hourly mean total suspended solids concentration as a function of time and measurement method.

\section{ACOUSTIC MEASUREMENTS}

\subsection{Doppler principle}

All ADVs and ADCPs work on the Doppler principle. In the beginning of a measurement cycle, an ultrasonic burst of a given frequency and with a fixed duration is emitted into the medium. At the end of the emission, the instrument switches in reception mode. The emitted signal travels along the beam axis and each encountered particle partly backscatters the acoustic wave. If the particle is moving in the medium, a frequency shift is observed in the backscattered wave (the so-called Doppler shift). This assumes that the velocity of the suspended particles is equal to the flow velocity. This working principle allows the precise knowledge of the position in the flow of a given velocity or backscattered signal amplitude at a given time stamp.

In the same time, due to thermal conduction and viscosity effects, the intensity of the ultrasonic wave propagating in a homogeneous medium decreases. In particle laden flows, an additional attenuation due to the scattering and the absorption by the particles themselves contribute to the intensity decay.

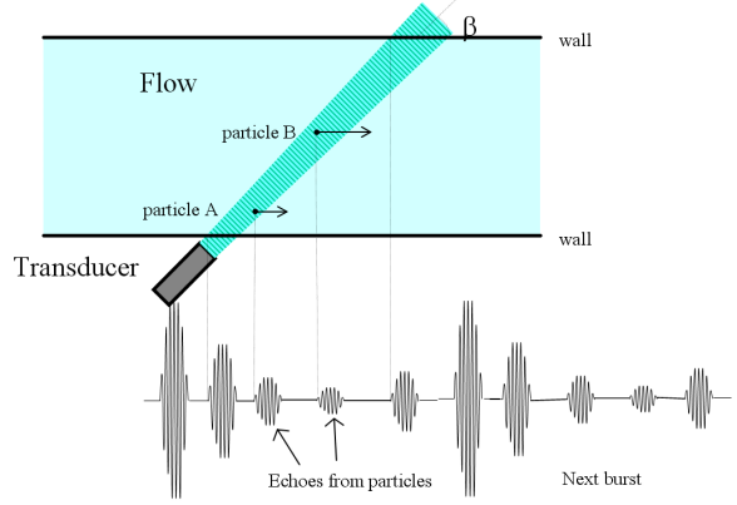

Figure 2 Pulsed Doppler Principle

The signal within each volume will be function of the number of suspended particles in the volume and their velocity. The Doppler signal frequency only depends on the particles velocity. Several methods are used to extract the Doppler information from the volume's backscattered signal. An estimator allows the calculation of the mean Doppler frequency $f_{\mathrm{D}}$, thus the flow velocity $V$, in the corresponding volume according to : 
$V=\frac{c \cdot f_{D}}{2 f_{0} \cdot \cos \beta}$

where $c$ is the speed of sound in the medium, $f_{0}$ the carrier frequency and $\beta$ the angle between the flow and the ultrasonic beam.

The velocity profile is obtained along the beam and its integration over the flow section gives the flow rate.

\subsection{Acoustic turbidity}

Turbidity is usually associated to the haziness of a fluid due to the light diffusion caused by the particles it contains. In the same way, the acoustic turbidity defines the modification of the ultrasonic beam induced by the presence of suspended sediments in the fluid. A simple approach consists to rely on the evolution of the backscattered signal amplitude to describe the acoustic turbidity.

\subsubsection{Theoretical expression}

On the theoretical point of view, for an acoustic flowmeter, the recorded root-mean-square of the backscattered voltage can be written (Thorne et al., 2002) at range $r$ as follows:

$V_{r m s}=\frac{k_{s} k_{t}}{r \psi} M^{1 / 2} e^{-2 \alpha r}$

with

$$
\alpha=\alpha_{w}+\alpha_{s}=\alpha_{w}+\frac{3}{4 \rho_{s} r} \int_{0}^{r} \frac{\chi_{m}}{\left\langle a_{s}\right\rangle} M\left(r^{\prime}\right) d r^{\prime}
$$

and

$$
\begin{aligned}
& k_{s}=\frac{\langle f\rangle}{\left(\rho_{s}\left\langle a_{s}\right\rangle\right)^{1 / 2}} \\
& k_{t}=R T_{v} P_{0} r_{0}\left\{\frac{3 \tau c}{16}\right\}^{1 / 2} \frac{0,96}{k a_{t}}
\end{aligned}
$$

$V_{\mathrm{rms}}$ is an average value over a large number of backscattered receptions. $\psi$ stands for the near field correction, $M$ is the particle concentration, $\alpha_{w}$ is the attenuation due to the water absorption and $\alpha_{\mathrm{s}}$ is the particle attenuation mainly due to scattering for non cohesive particles insonified at megahertz ultrasound frequencies. As shown in (3), $\alpha_{\mathrm{s}}$ is related to the normalized total scattering cross-section $X_{\mathrm{m}}$ of the particle.

$k_{\mathrm{s}}$ represents the particle backscattering properties, with $\langle f>$ the averaged form function which describes the backscattering characteristics of the particles, $\rho_{\mathrm{s}}$ the particle density, $\left\langle a_{\mathrm{s}}\right\rangle$ the mean particle radius.

$k_{\mathrm{t}}$ is an acquisition system constant for a given setting. $P_{0}$ is the reference pressure, normally defined at $r_{0}=1 \mathrm{~m}, R$ is the receiver sensitivity, $\mathrm{T}_{\mathrm{v}}$ is the voltage transfer function of the system, $\tau$ is the pulse duration, $c$ is the velocity of sound in water, $k$ is the wavenumber for sound in water ( $k=2 \pi / \lambda, \lambda$ is the wavelength of sound in water), and $a_{\mathrm{t}}$ is the radius of the transducer. 
Given equation (2), the exact expression of the acoustic turbidity would be:

$T=\frac{V_{r m s} r \psi}{k_{t}} e^{\alpha_{w} r}=k_{s} M^{1 / 2} e^{-2 \alpha_{s} r}$

In equation (6), the right part only depends on the particle characteristics. The attenuation of water, $\alpha_{w}$, is function of the frequency and the water characteristics (temperature, salinity and pressure). Its value can be calculated from a semi-empirical formula (Fisher et al. 1977).

$\alpha_{w}=\left(55.9-2.37 T+4.77 \cdot 10^{-2} T^{2}-3.48 \cdot 10^{-4} T^{3}\right) \cdot 10^{-15} F^{2}$

$T$ is the temperature in Celsius degrees and $F$ is the frequency in $\mathrm{Hz}$.

By comparing equations (2) and (6), one can see that at a fixed distance $r$, the backscattered amplitude and the acoustic turbidity will show the same variations. We will therefore use the backscattered amplitude as first approach of the acoustic turbidity.

\subsubsection{Interpretation}

As shown previously, the backscattered signal directly includes information about the particles encountered in the explored medium. With adequate analysis, different elements concerning the nature and the concentration of the particles can be extrapolated. If the particles in the medium are well known, in terms of shape, size and density, their acoustic characteristics can be determined. If the content of the flow is unknown, only a qualitative interpretation can be made as the relative behaviour of SSC for example.

Scattering loss, which is due to the reflection of the incident acoustic energy, is a function of the ratio of the acoustic wavelength, $\lambda$, to particle circumference $2 \pi a_{s}$. Let $x$ be $x=k<a_{s}>$ where $a_{s}$ is the mean particle radius.

For $x \ll 1$, the Rayleigh regime, the wavelength of sound is much greater than the particle circumference and thus the scattering is considered to be independent of the particle shape. In this case, the scattered energy is partly reflected back toward the source, and the scattering losses rise rapidly with increasing sediment size.

For $x>>1$, the geometric regime, the wavelength of sound is smaller than the particle circumference, and the scattering cross-section is directly related to the particle's geometry. Half of the scattering propagates in the forward direction and the remainder scatters through all directions.

As $\lambda$ approaches $2 \pi a_{\mathrm{s}}$ scattering becomes complex and changes rapidly with sediment size and frequency (Urick, 1948). Because of this physical relation between frequency and sediment size, there will be an optimal acoustic frequency for a given particle size distribution associated to the case $x=1$. Therefore, if the smallest particle to be measured has a radius $a_{\min }$, with $c$ the speed of sound, the optimal choice of frequency $F$ would be:

$$
F=\frac{c}{2 \pi a_{\text {min }}}
$$

The particles with smaller radius will have a negligible contribution to backscattering.

As compiled in a review paper (Landers 2010), different methods can be used to estimate the SSC. Three treatment philosophies can be distinguished: the oldest one, developed by Urick which gives the sonar equation (Urick 1948); the semi-empirical method, equation (2), 
developed and complemented by many researchers (Sheng et al. 1988, Thorne et al. 2002); and a mixed method, using attenuation and backscattering developed by Topping (Topping et al. 2007).

\section{EXPERIMENTAL RESULTS}

\subsection{Instrumentation}

All the following measurements where done with an UB-Flow ADV. It is equipped with two wideband transducers centred on 1.5 and $6 \mathrm{MHz}$ respectively emitting at 75 and $55^{\circ}$ regarding to the instrument's base.

Multiple settings are possible. The emission frequencies and the pulse repetition frequency as well as the cell depth, the number of cells and the inter-cell distance are adjusted by the user. Also the number of samples used in a cell to evaluate the velocity and the amplitude value is fixed by the user. Gain is manually fixed or automatically adjusted.

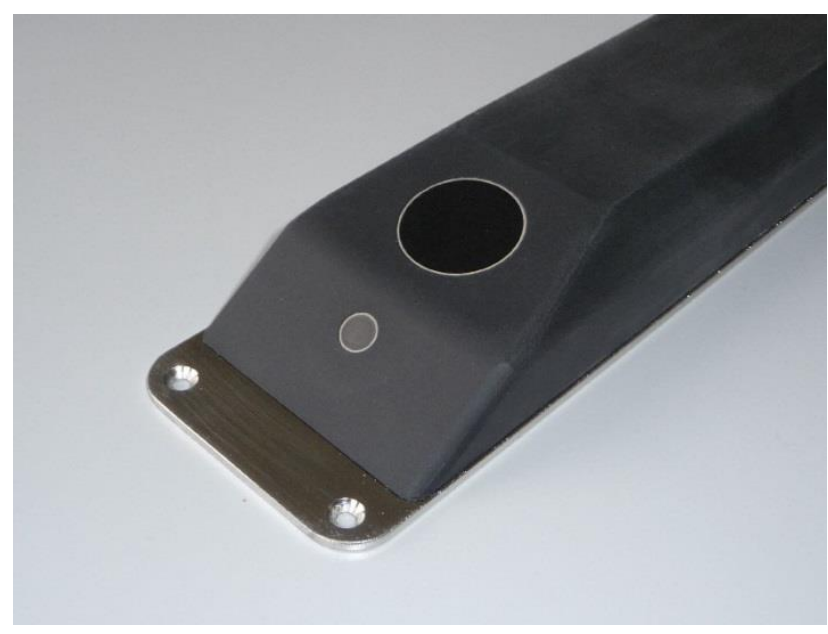

Figure 3 An UB-Flow Acoustic Doppler Velocimeter.

For each cell and each time stamp, backscattered amplitude and velocity are recorded. No data treatment was applied to the following amplitude data. It could be roughly linked to sediment concentration after adequate calibration or give more precise concentration values by using the data inversions techniques mentioned above (Landers 2010).

\subsection{Aar River}

Some measurements were done in the urban Aar River (figure 4). The instrument was placed on the river bottom and was emitting upwards at $1.5 \mathrm{MHz}$. This frequency is commonly used to monitor velocities and corresponds to the optimal frequency for particles of $160 \mu \mathrm{m}$ radius.

Figure 5 shows a typical backscattered amplitude profile. The first peak and the values below correspond to the signal in the near field zone of the transducer and can not be exploited without correction. Afterwards, the amplitude decreases with depth, which is the expected behaviour due to attenuation and spherical spreading of the beam. 


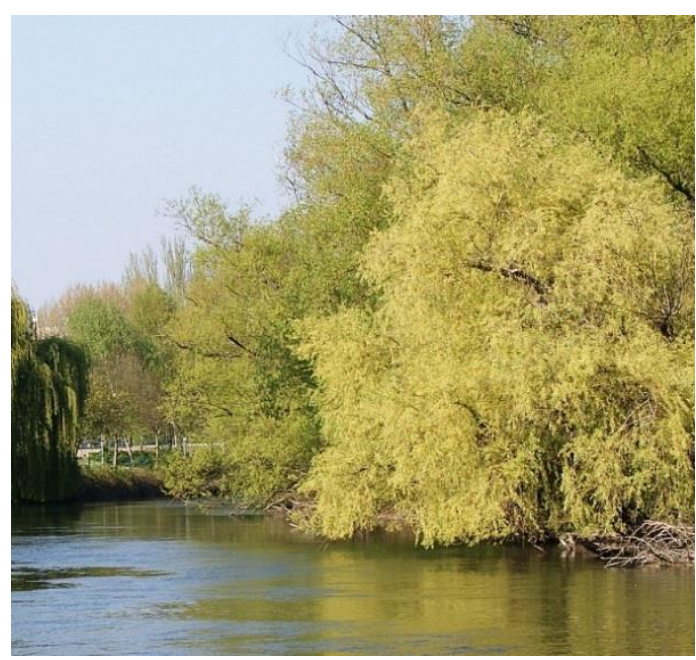

Figure 4 A bucolic glimpse on the Aar River.

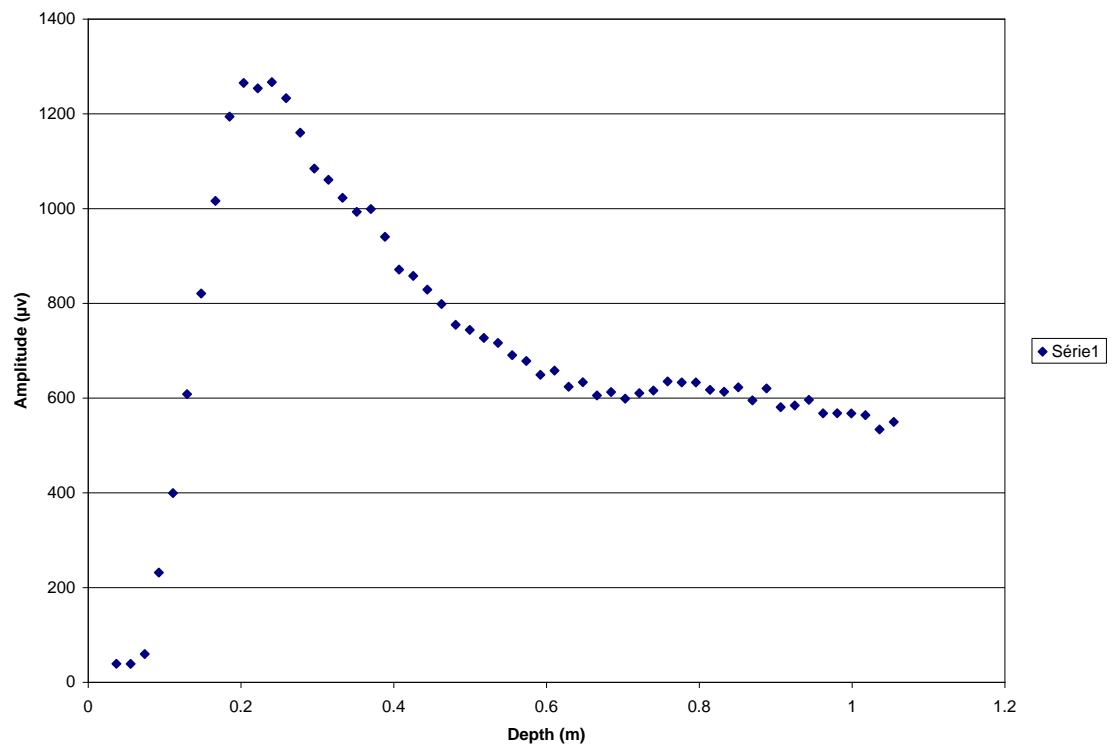

Figure 5 Backscattered amplitude evolution with depth.

An example of qualitative measurement is given figure 6 . It shows the temporal behaviour of the backscattered amplitude in a given measurement cell. The first set of data corresponds to dry weather conditions: the global amplitude is low with some peaks identified as bigger particles traveling through the measurement cell. The second set was obtained during a rain event. The wash-off phenomena has a direct consequence on the signal: a clear increase of amplitude is due to the increase of particles in the fluid. 


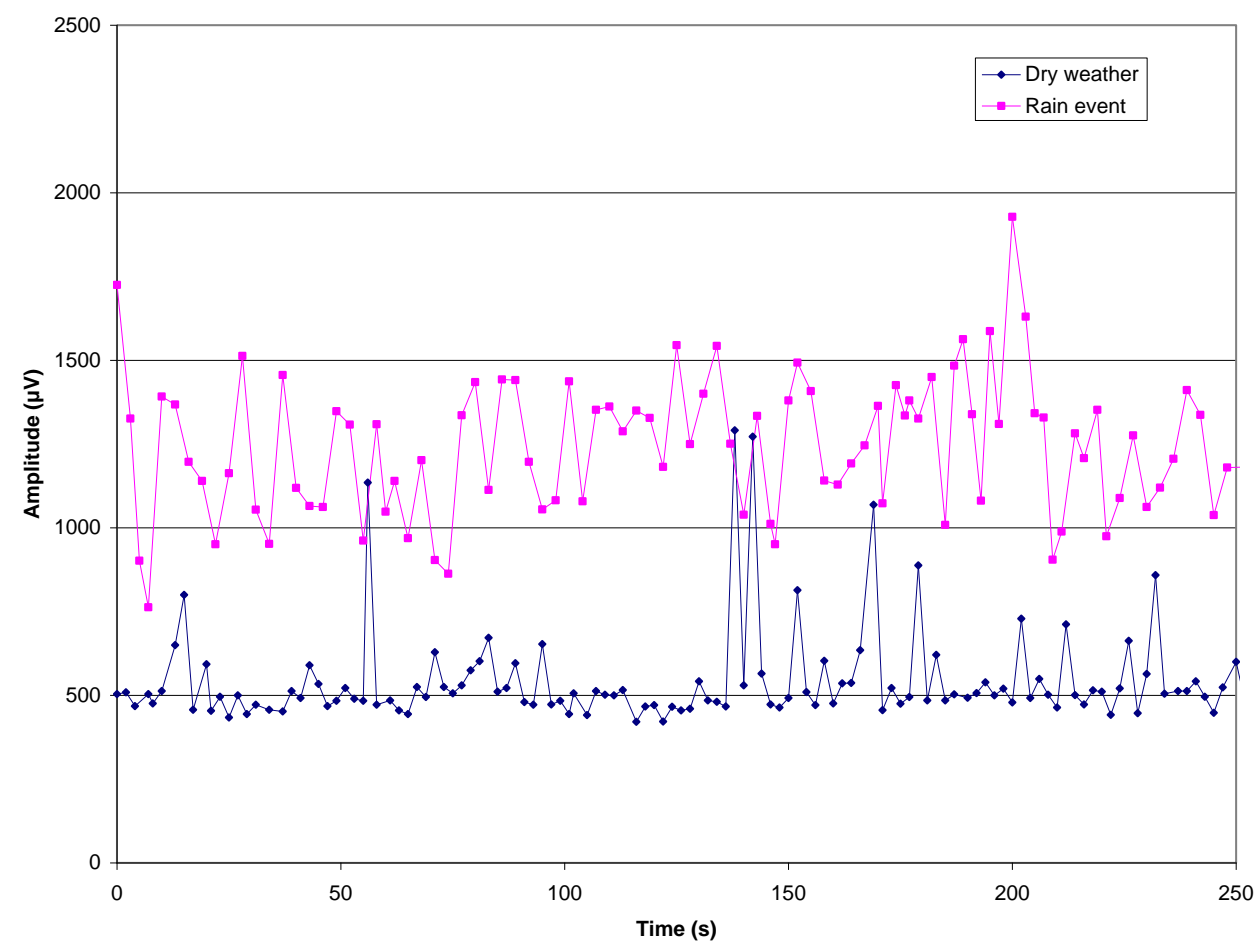

Figure 6 Backscatter amplitude time evolution in a given measurement window.

Thus, the backscattered amplitude could be a reliable concentration indicator. It could, for example, be used to monitor jointly the suspended sediment flow in a river, with its concentration and velocity characteristics.

\subsection{Couesnon River}

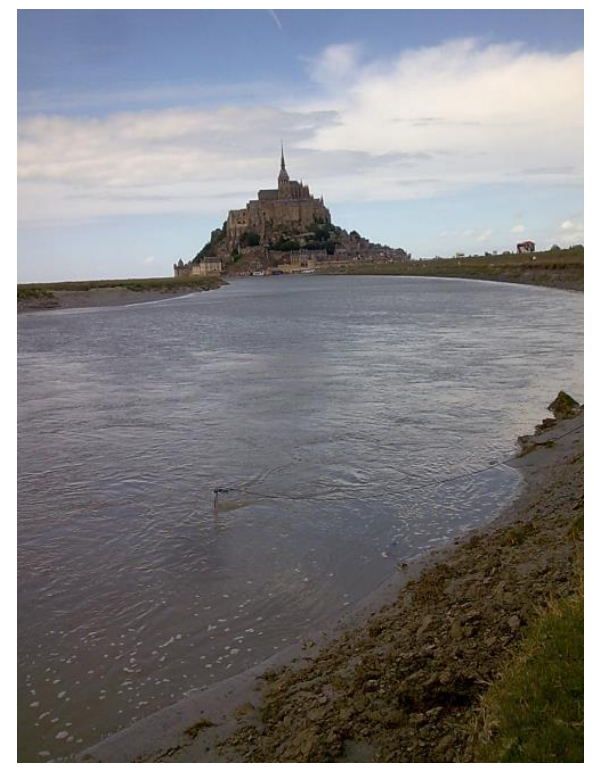

Figure 7 The Couesnon River with St Michel Mount in background. 
This second measurement campaign shows more pertinent analysis still without acoustic amplitude inversion. The Couesnon River is located in the bay of St Michel's Mount (figure 7). It is daily flushed to maintain the insular character of the Mount.

The instrument was fixed on a support, looking downwards to the bottom of the river, emitting at 6.25 MHz. At this frequency, the ultrasonic beam is mostly sensitive to fine particles with a radius of $40 \mu \mathrm{m}$. The bottom appears as a peak at $17 \mathrm{~cm}$ on figure 8 . This figure also shows the temporal evolution of the backscattered amplitude profile. The mentioned time is the time delay between the beginning of the flush and the observation. As expected, the amplitude increases with time, resulting from the increasing sediment concentration in suspension in the flow.

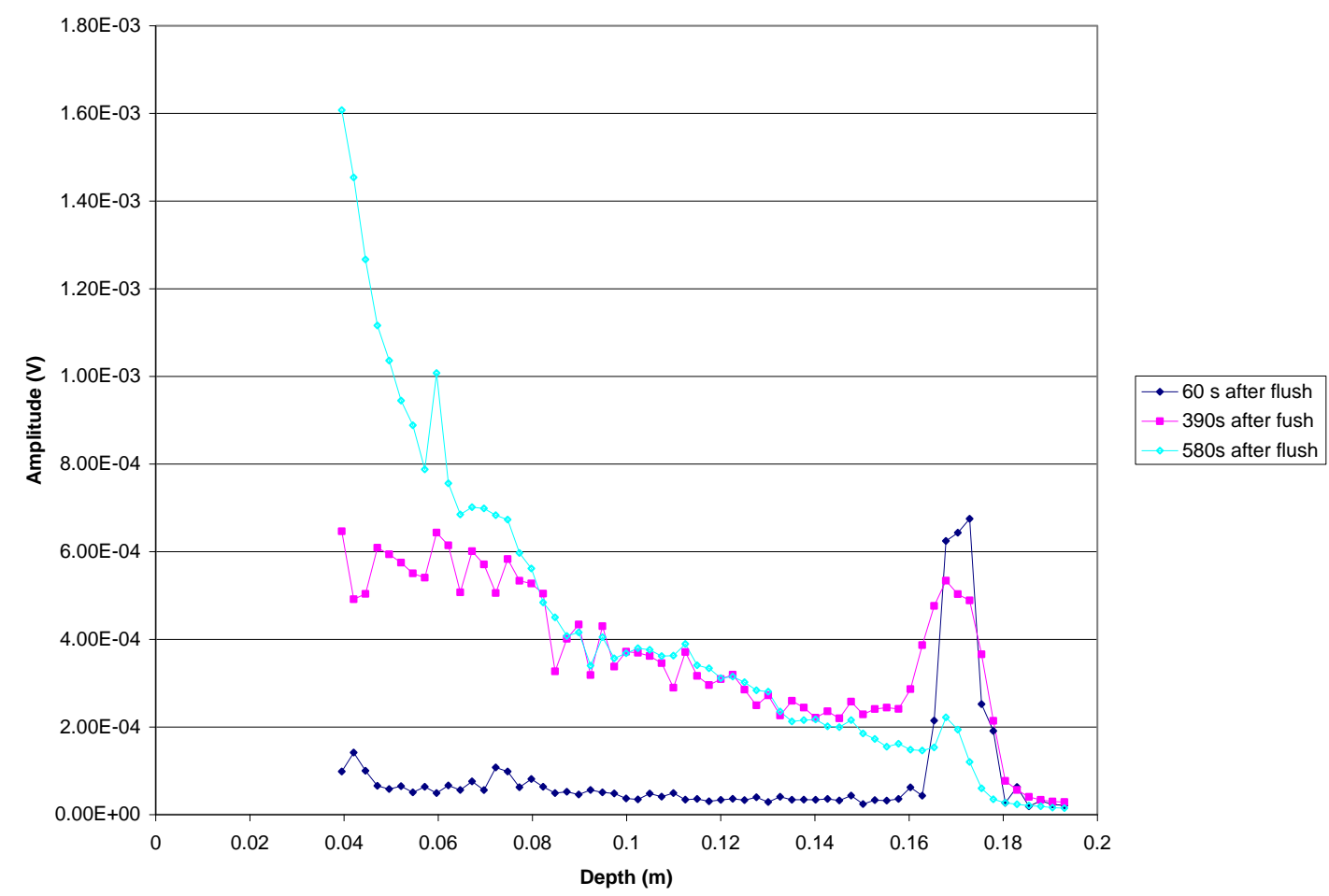

Figure 8 Backscattered amplitude evolution with depth and time delay with the beginning of the flush.

Amplitude and velocity data can easily be compared as shown in figure 9. It shows the values measured in the same cell, situated $5 \mathrm{~cm}$ away from the instrument. As mentioned earlier, one can see the increase of velocity with time after the beginning of the flush. After about 300 seconds, the velocity value becomes unsteady, revealing a turbulent regime of the flow. A good correlation with the amplitude behaviour can be noticed: at the same time the backscattered amplitude begins to increase significantly. A threshold velocity can thus be associated the sediment suspension.

This simple analysis suggests that some interesting results on sediment transport could easily be obtained by the combined analysis of turbidity and velocity data. 


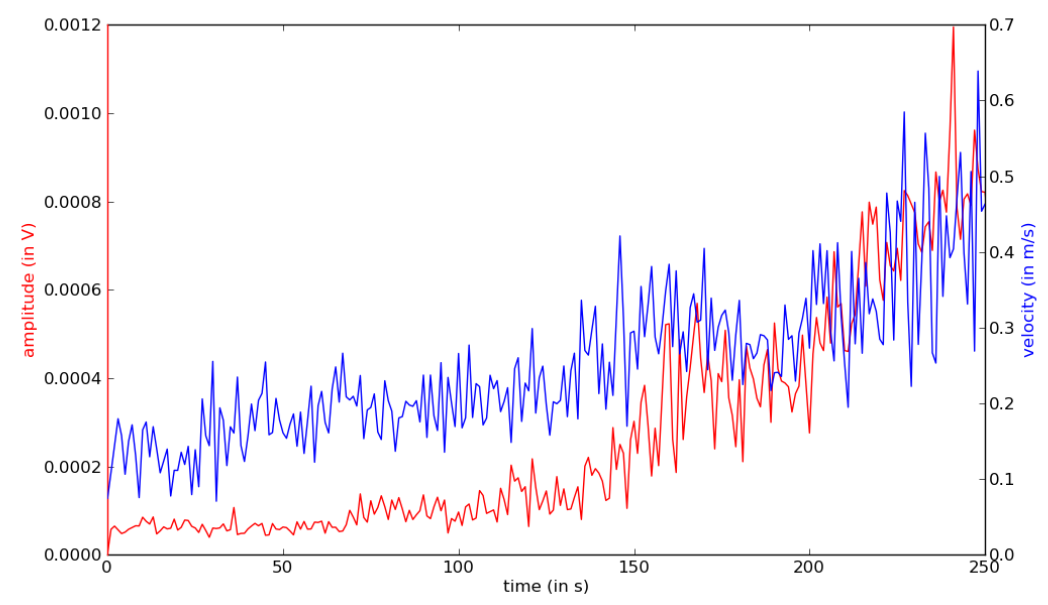

Figure 9 Temporal evolution of velocity and backscattered amplitude.

\section{CONCLUSIONS}

It is clear that ADVs and ADCPs are robust instruments but their signal output must be processed carefully. As shown above, simple experiments with an unique frequency and no complicated data treatment, can already bring interesting results. With complementary frequency screening, additional information on the size distribution of the particles could be obtained.

This explains the growing success of ADCPs, already used for qualitative studies of suspended sediments in rivers for years. More recently ADCPs are used for horizontal profiling. A recent paper (Moore et al. 2012) shows an application of H-ADCPs for the quantitative monitoring of suspended sediment concentration. Excellent agreement with turbidity and sampling data has been observed.

Two levels of use of acoustic devices in rivers could be distinguished. A macroscopic use of the acoustic data could be identified as river gauging. A finer analysis, corresponding to a microscopic use, could be associated to sediment transport analysis. Anyhow, with a good knowledge of the theoretical fundamentals, acoustic profiling is a powerful and promising technique.

\section{REFERENCES}

Chanson H., Takeuchi M., Trevethan M. (2008) Using turbidity and acoustic backscatter intensity as surrogate measures of suspended sediment concentration in a small subtropical estuary, J. Env. Man. 88, 1406-1416.

Downing, J. (2006) Twenty-five years with OBS sensors: The good, the bad, and the ugly, Cont. Shelf Res. 26, 2299-2318.

Fisher, F.H., Simmons, V.P. (1977), Sound ,absorption in sea water. Journal of the Acoustical Society of America 62,558-564.

Gray J.R, Gartner J.W. (2009) Technological advances in suspended sediment surrogate monitoring, Water Resources Research 45, 1-20. 
Hawley N. (2004) A Comparison of Suspended Sediment Concentrations Measured by Acoustic and Optical Sensors, J. Great Lakes Res. 30, 301-309.

Landers M.N. (2010) Review of methods to estimate fluvial suspended sediment characteristics from acoustic surrogate metrics, Proceedings of the $2^{\text {nd }}$ Joint Federal Interagency Conference.

Pallarès A., François P., Pons, M-.N., Schmitt P.: Suspended particles in wastewater: their optical, sedimentation and acoustical characterization and modeling, Water Science \& Technology 63.2 (2011), 240-247.

Sheng, J. and Hay, A. E. (1988), An examination of the spherical scatter approximation in aqueous suspensions of sand. J. Acoust. Soc. Am., 83(2), 598-610.

Thorne, P.D. and Hanes, D.M. (2002), A review of acoustic measurement of small-scale sediment processes, Continental Shelf Research, 22, 603-632.

Topping, D. J., Wright, S. A., Melis, T. S. and Rubin, D. M. (2007), High-resolution measurement of suspended sediment concentrations and grain size in the Colorado River in Grand Canyon using a multi-frequency acoustic system, in Proceedings of the 10th International Symposium on River Sedimentation.

Urick, R.J. (1948), The absorption of sound in suspensions of irregular particles, Acoustical Soc. of America, 20, 283-289.

Vousdoukas M.I., Aleksiadis S., Grenz C., Verney R., (2011) Comparisons of acoustic and optical sensors for suspended sediment concentration measurements under non-homogeneous solutions, Journal of Coastal Research 64, $160-164$. 


\section{ACOUSTIC TURBIDITY AS ONLINE MONITORING TOOL FOR RIVERS AND SEWER NETWORKS}

\section{Anne Pallarès ${ }^{1}$}

Philippe Schmitt ${ }^{1}$, Stéphane Fischer ${ }^{2}$

\footnotetext{
${ }^{1}$ Laboratoire lcube, Département de Mécanique, Strasbourg ${ }^{2}$ UBERTONE, Strasbourg
} 


\section{Outline}

- Working context

- Pulsed ultrasound

- Incoherent scattering

- Material and methods

- Experimental results

- Conclusions 


\section{Our primary working context}

- Wastewater management: Online Water Quality Monitoring

- Real time flow control

- Complex medium with variable characteristics

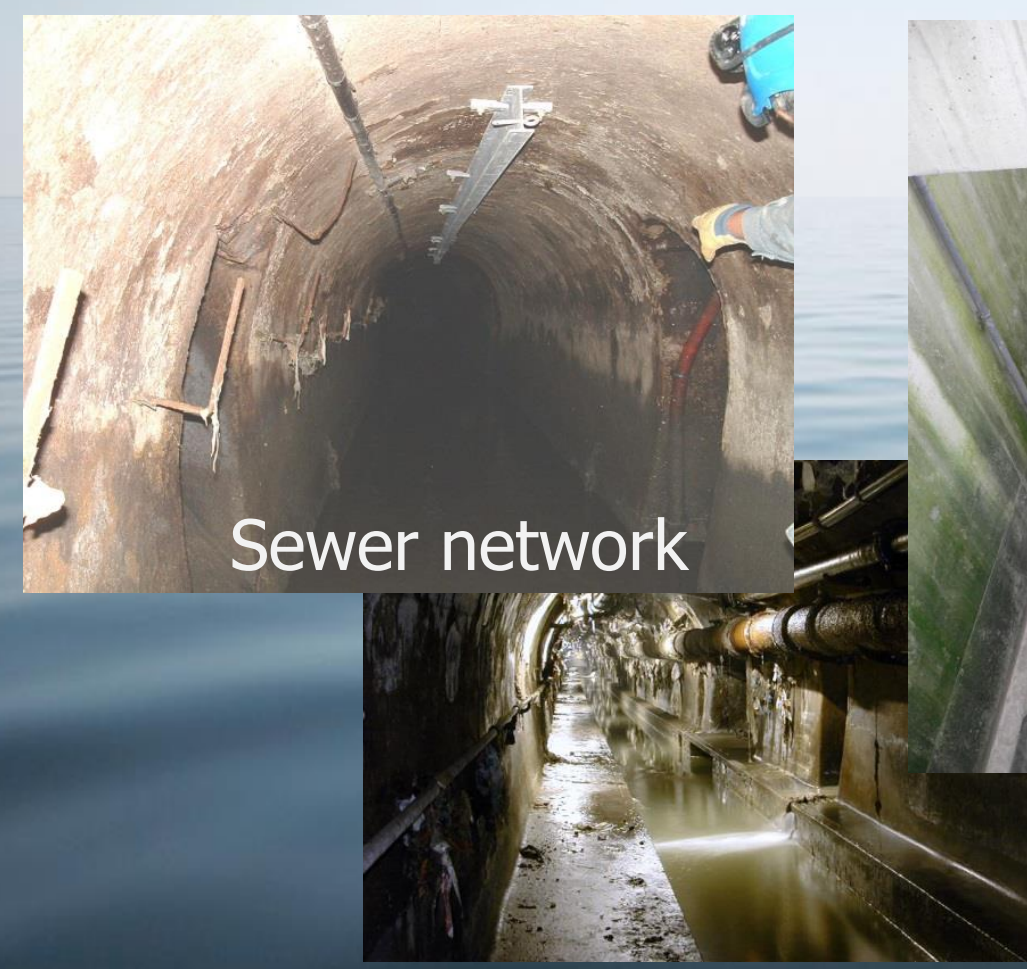

Karlsruher Flussgebietstage 2013,

20-21 june

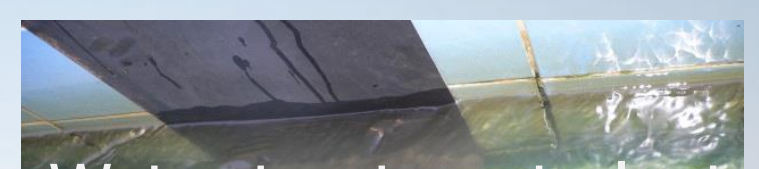

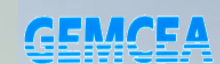

Water treatment plant

CNrS
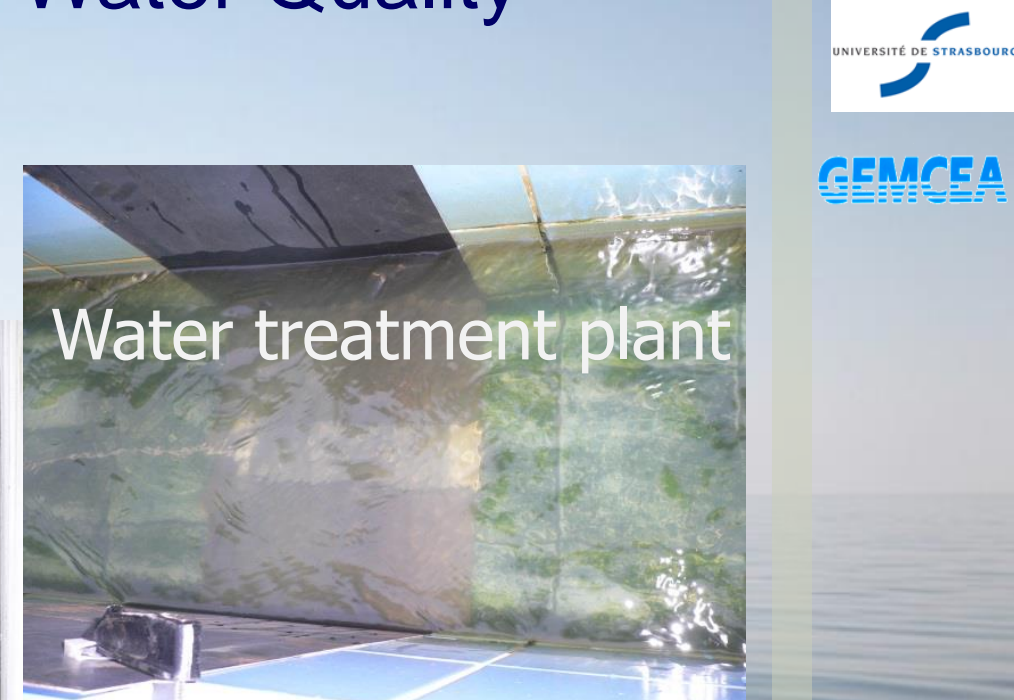

Sewer network

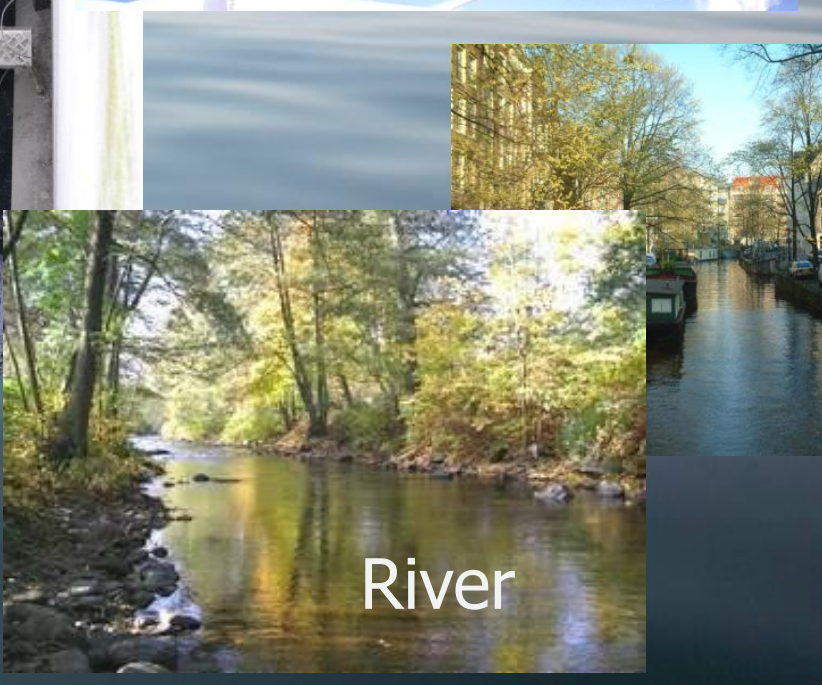




\section{How to monitor sediments in water?}

- Sampling

- Time dependant sample collection

- Temporal sample evolution

- Filtered on $1 \mu \mathrm{m}$ filter

- Total suspended solid concentration calculated from the solid deposit

$\Rightarrow>$ time delay forbids any real time retroaction
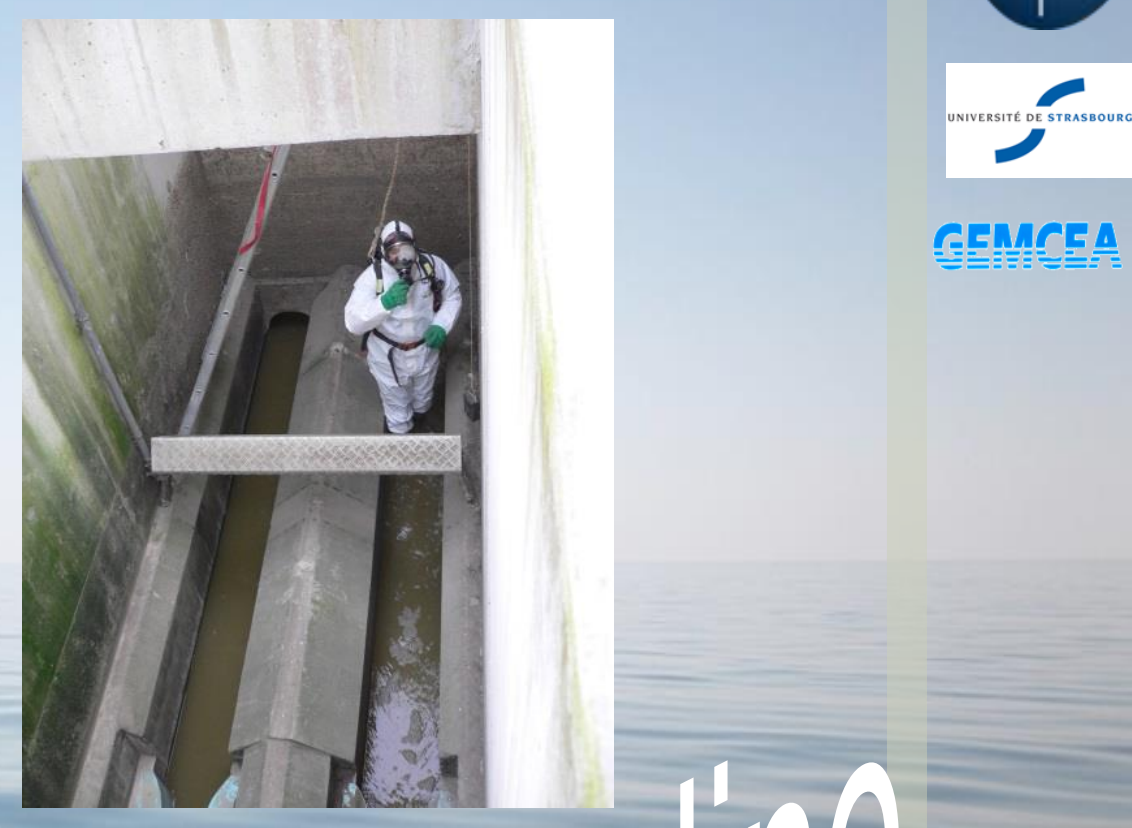

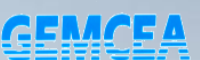

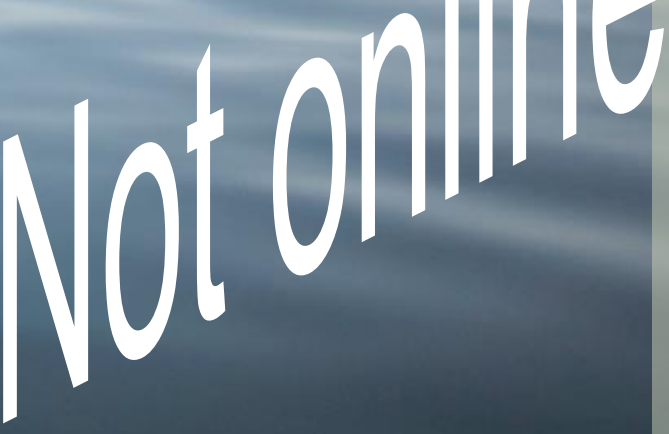




\section{How to monitor sediments in water?}

- Turbidity

- Sensitive to installation location

- Noisy device if presence of large particles

- Sensitive to the presence of dye

- Needs site calibration

$\Rightarrow$ several factors leads to a change of the turbidity/concentration relationship
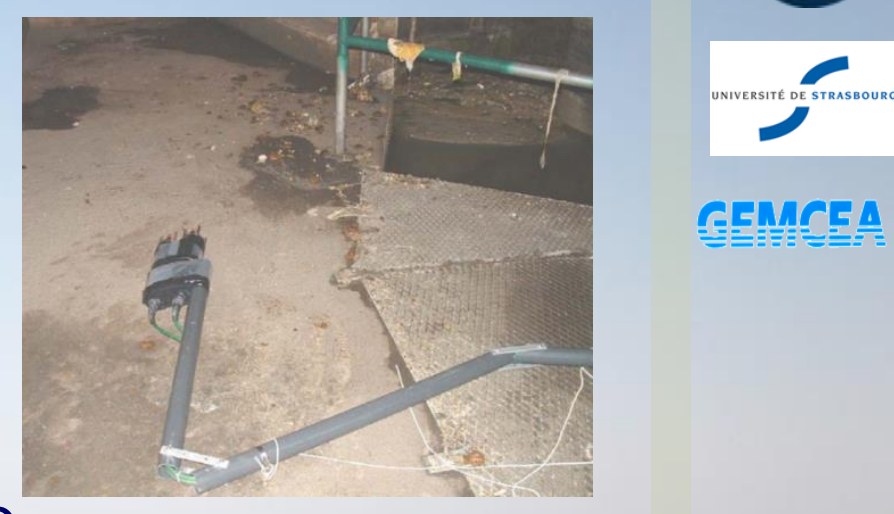

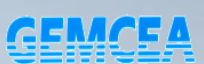

- Acoustics

- Huge variety of materials in various proportions

o wastewater modeling

- Mineral and organic compounds

- Size distribution

$\Rightarrow>$ reliable indicators of the total suspended solids less as possible calibration steps 


\section{Pulsed Ultrasound - Scattering}

Sound pulse

Emission

Transducer

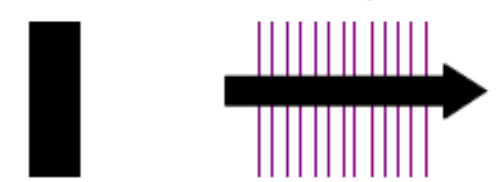

Reception

Transducer
Scatterers

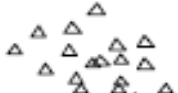

$\Delta \Delta \Delta_{\Delta}^{\Delta} \Delta_{\Delta}^{\Delta}$

$\Delta \Delta_{\Delta \Delta}^{\Delta \Delta}$
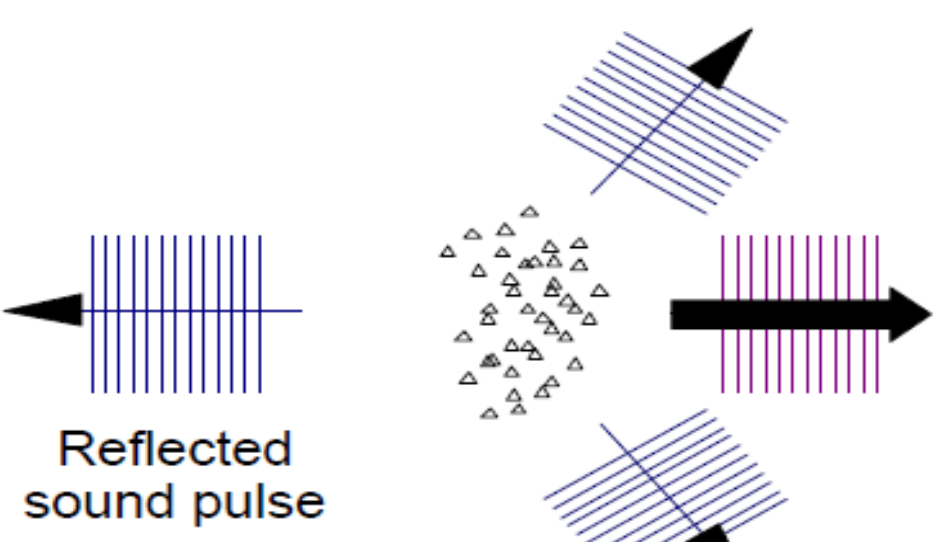

름ㅁ드를 


\section{Pulsed Ultrasound - Doppler Principle}

transmitted pulse $F_{o}$

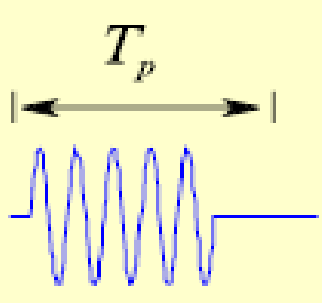

ionTek, Inc

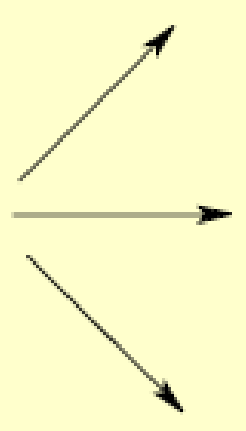

stationary or moving across

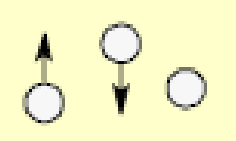

$\leftarrow 0$

moving away

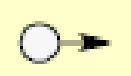

received signal $F_{D}$

target moving towards

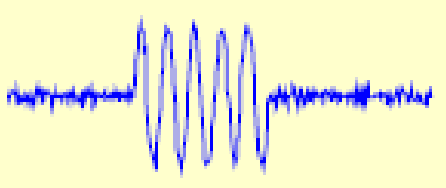

$F_{D}>F_{0}$

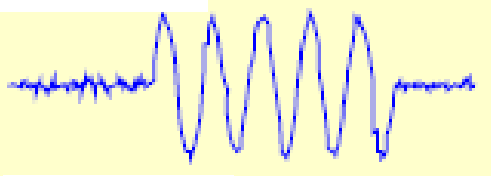

$F_{D}<F_{0}$

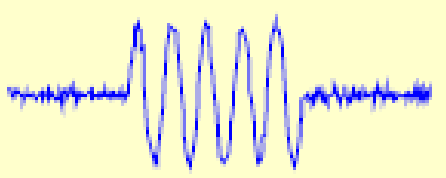

$F_{D}=F_{0}$

Karlsruher Flussgebietstage 2013,

20-21 June 


\section{Pulsed Ultrasound - Working principle}

TICU3E

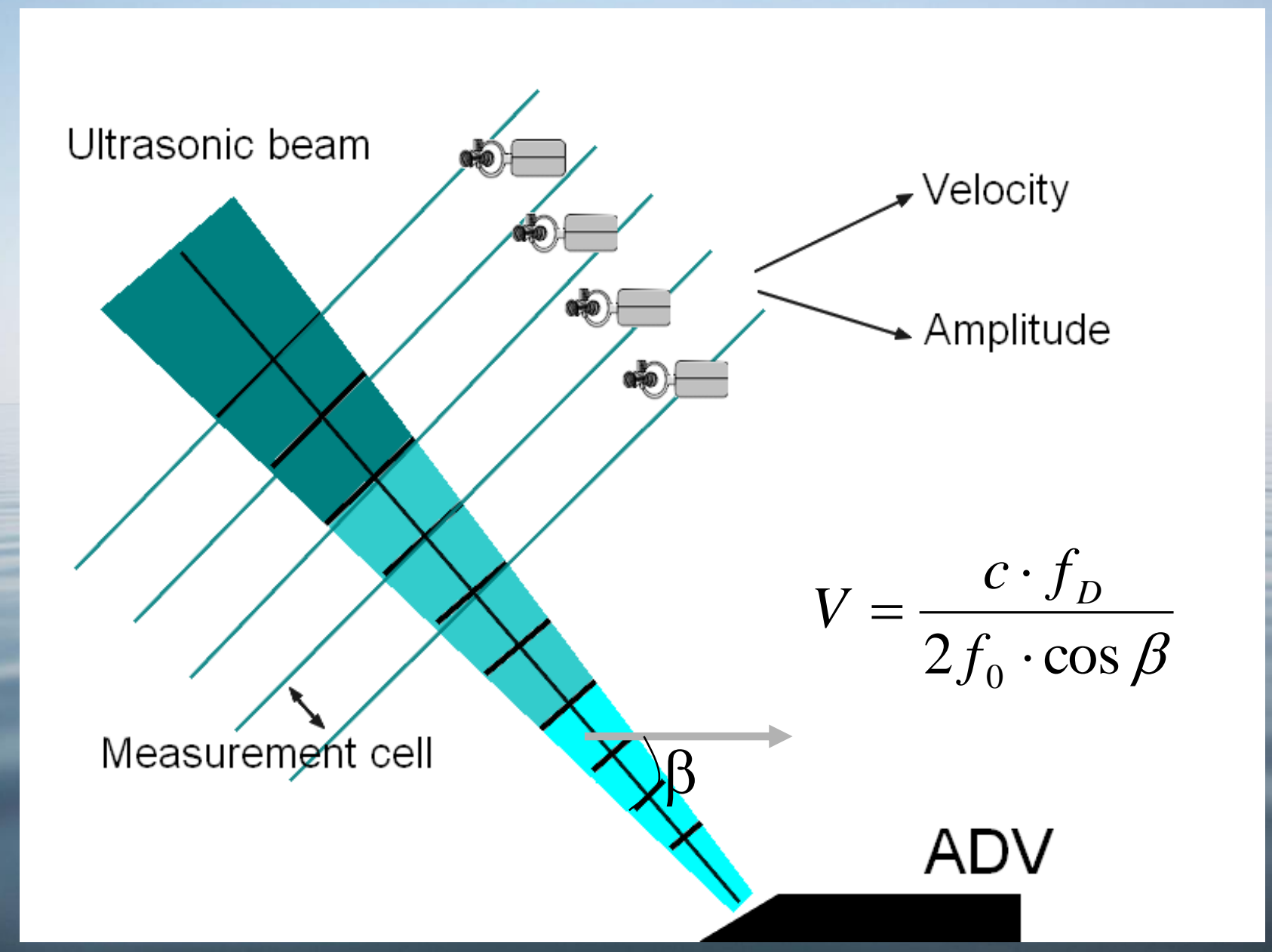

Karlsruher Flussgebietstage 2013, 


\section{Acoustic incoherent scattering}

- Root mean square voltage of the backscattered signal

$$
V_{r m s}=\frac{k_{s} k_{t}}{r \psi} M^{1 / 2} e^{-2 \alpha r}
$$

- $\quad r$ : distance along the beam ( $m$ )

- $\mathrm{k}_{\mathrm{t}}$ : system constant for a given setting

- $\mathrm{k}_{\mathrm{s}}$ : sediment backscattering properties

○ $\psi$ : near field correction

- M: sediment concentration

- $\alpha$ : attenuation of the medium 


\section{Acoustic incoherent scattering}

- $\mathrm{k}_{\mathrm{t}}$ : system constant for a given setting

$$
k_{t}=R T_{v} P_{0} r_{0}\left\{\frac{3 \tau c}{16}\right\}^{1 / 2} \frac{0,96}{k a_{t}}
$$

- $\mathrm{k}_{\mathrm{s}}$ : sediment backscattering properties

$$
k_{s}=\frac{\langle f\rangle}{\left(\rho_{s}\left\langle a_{s}\right\rangle\right)^{1 / 2}}
$$

- $<f>$ : averaged form function describing the backscattering characteristics of the particles 


\section{Acoustic incoherent scattering}

- $\alpha$ : attenuation

$$
\alpha=\alpha_{w}+\alpha_{s}=\alpha_{w}+\frac{3 \chi_{m} M}{4 \rho_{s}\left\langle a_{s}\right\rangle}
$$

- $\chi_{\mathrm{m}}$ : normalised total scattering cross-section (worth 1 for a rigid sphere in geometrical regime) 


\section{Acoustic incoherent scattering}

- Expression of acoustic turbidity

$$
T=\frac{V_{r m s} r \psi}{k_{t}} e^{2 \alpha_{w} r}=k_{s} M^{1 / 2} e^{-2 \alpha_{s} r}
$$

- Backscattered amplitude and the acoustic turbidity will show the same variations at given $r$

- Backscattered amplitude = first approach of the acoustic turbidity

- No data inversion wanted 


\section{Field instrument}

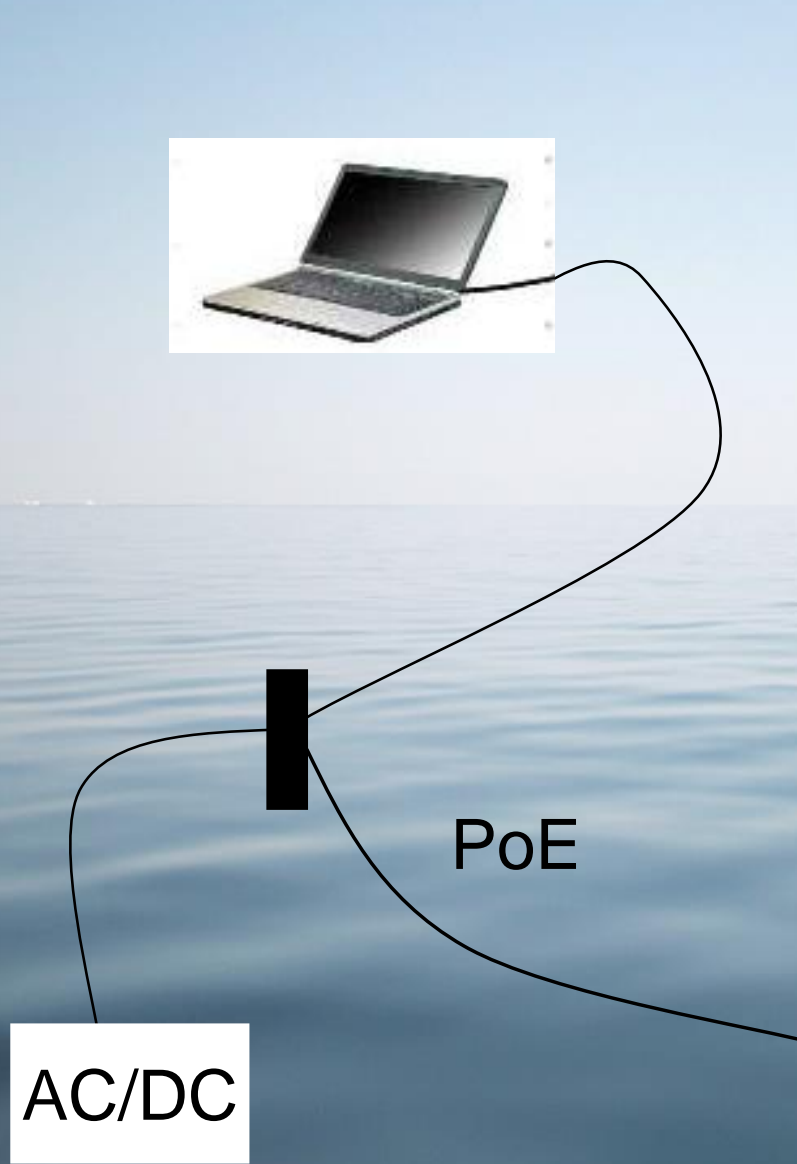

Karlsruher Flussgebietstage 2013,

20-21 June

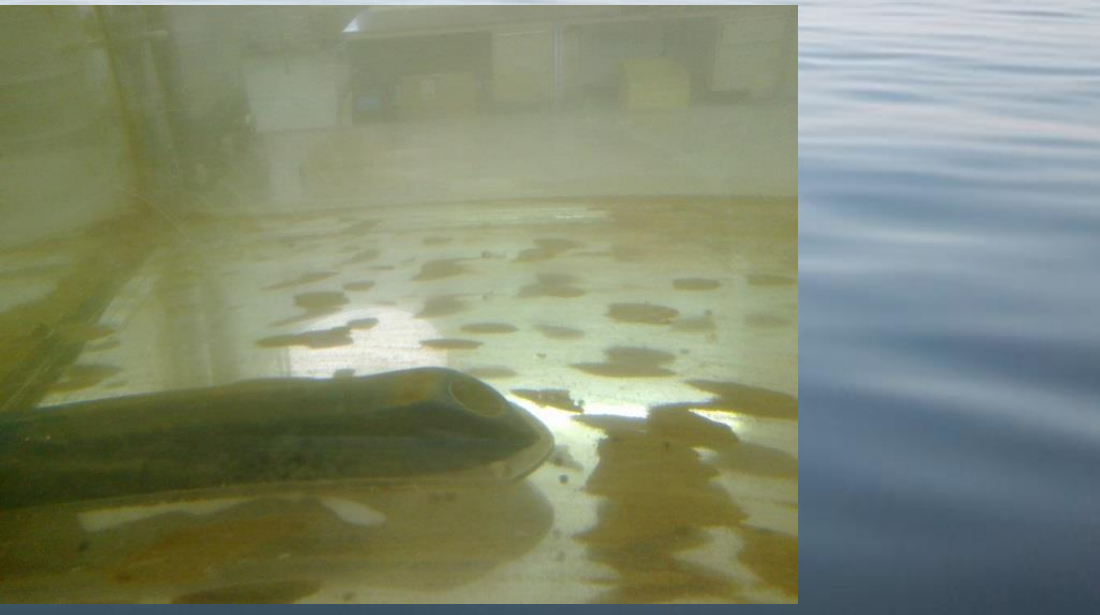

Submersible frame

\section{Web interface}

TiciJ3E

CNIS

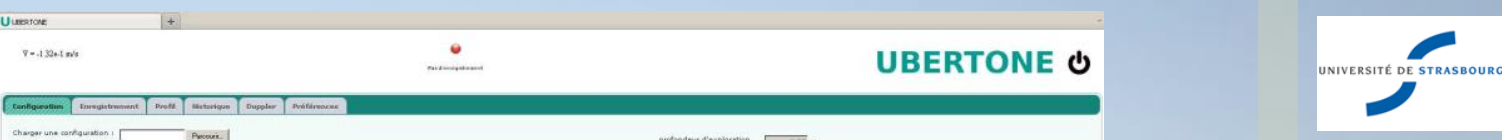

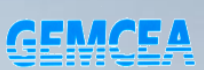




\section{Typical amplitude profile}

- No near field correction on amplitude

- Only onsidering the decreasing exponential part

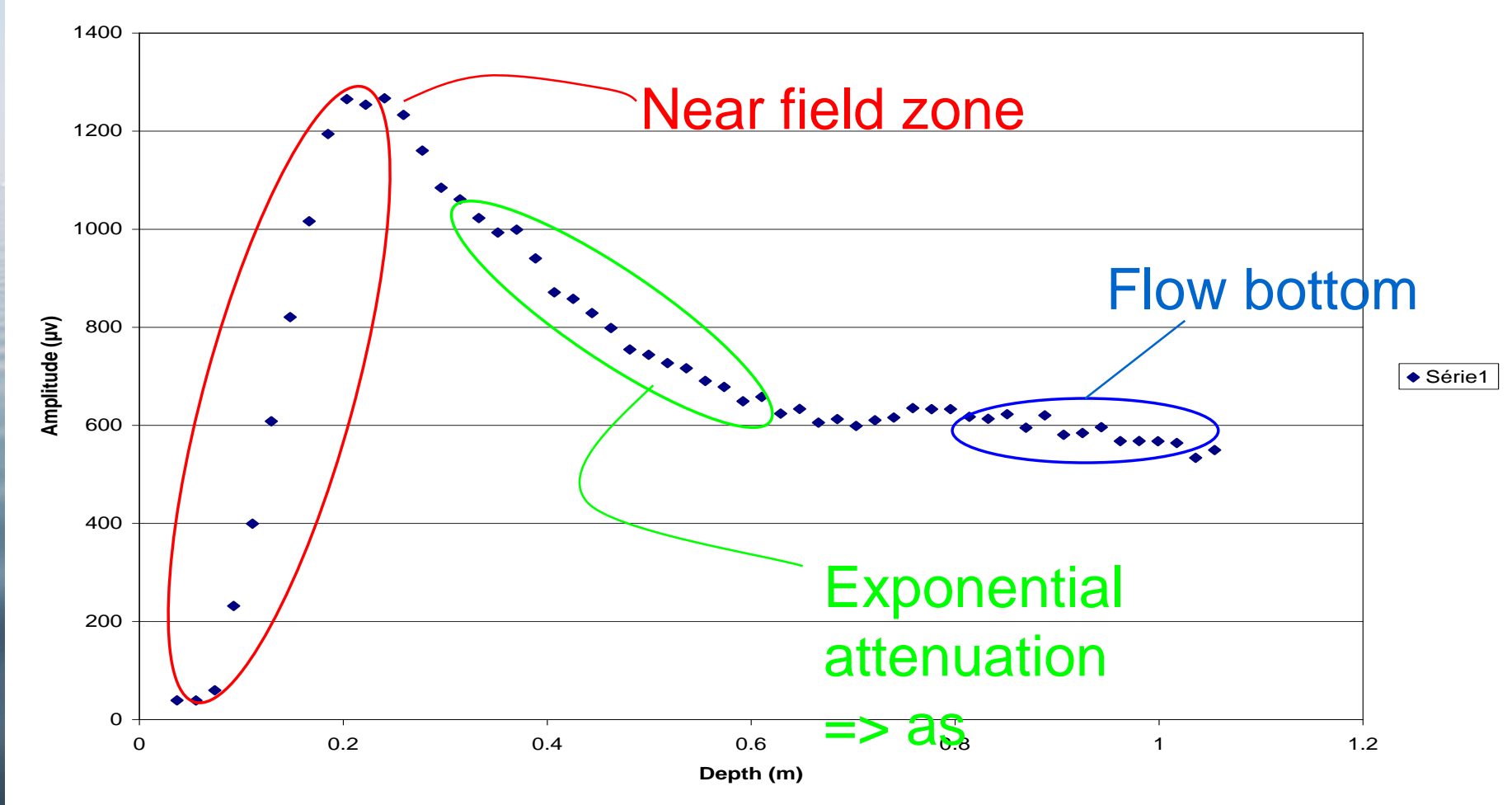

Karlsruher Flussgebietstage 2013, 


\section{Aar River}

TICIJE
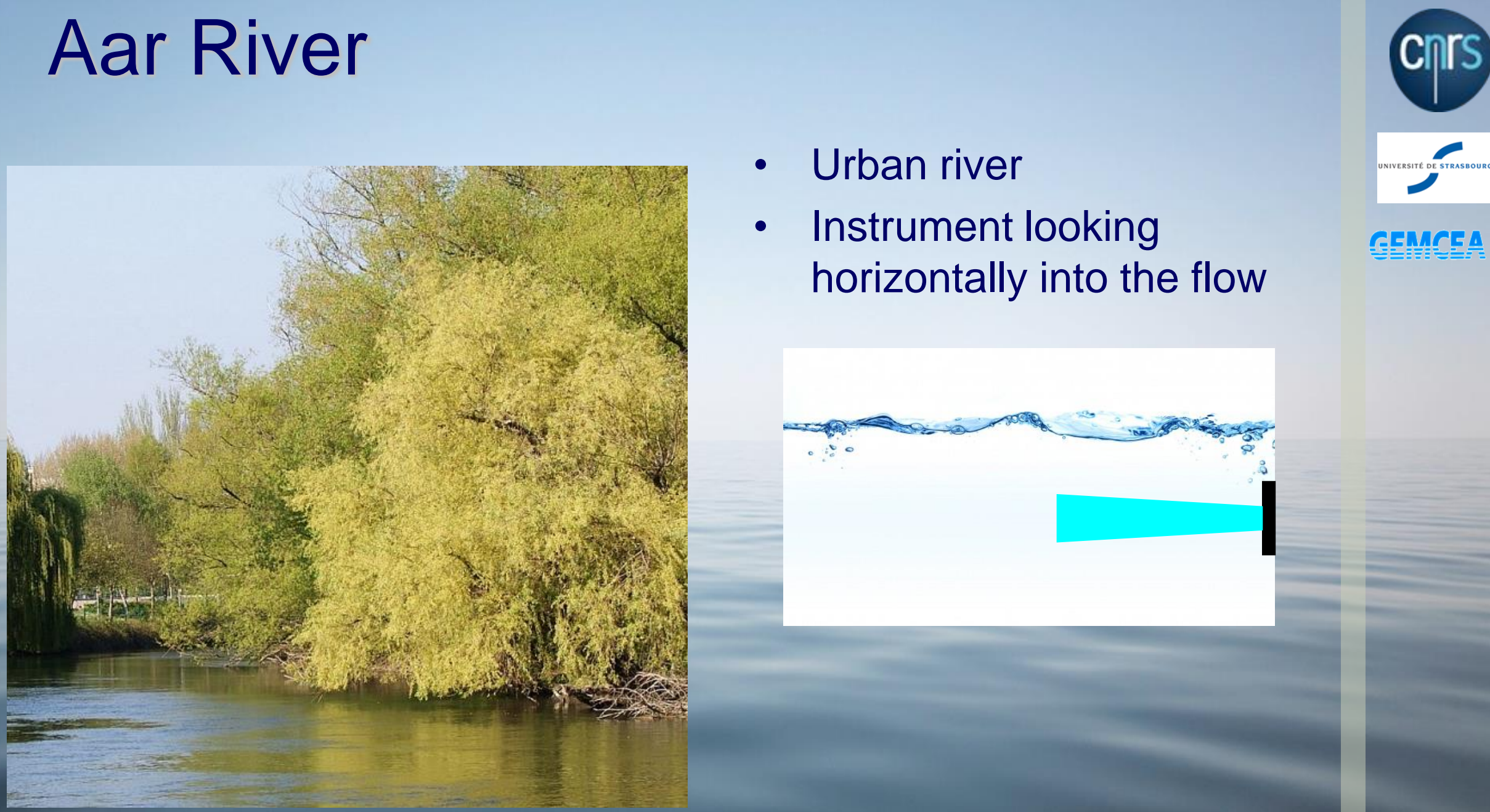

- Urban river

- Instrument looking horizontally into the flow

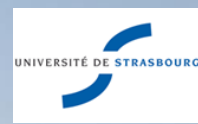

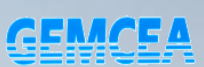

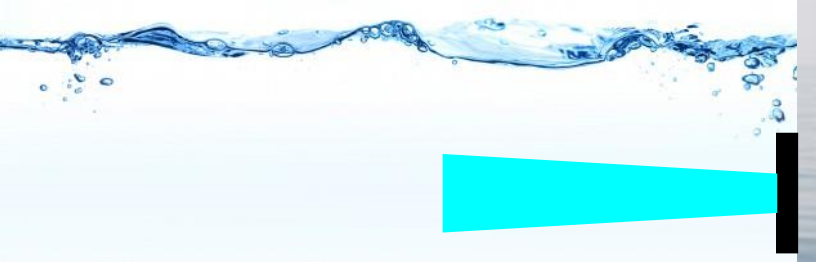

Karlsruher Flussgebietstage 2013,

20-21 June 


\section{Aar River}

CथrS

- Amplitude measurement at 1.5 MHz as a function of time and weather

- Increase of amplitude due to the wash-off particles

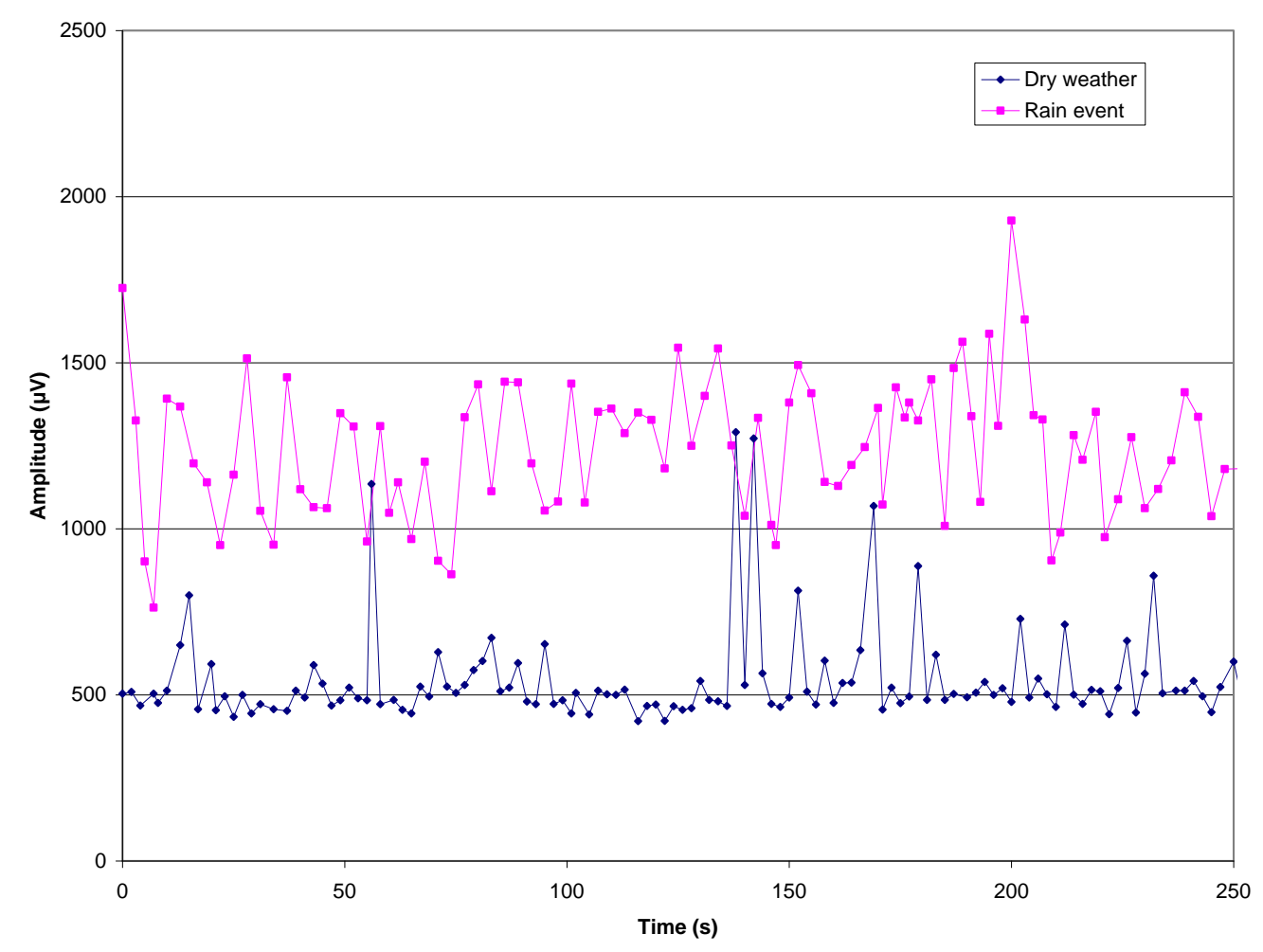




\section{Couesnon}

- River flushed every day

- Instrument looking downwards to the flow bottom
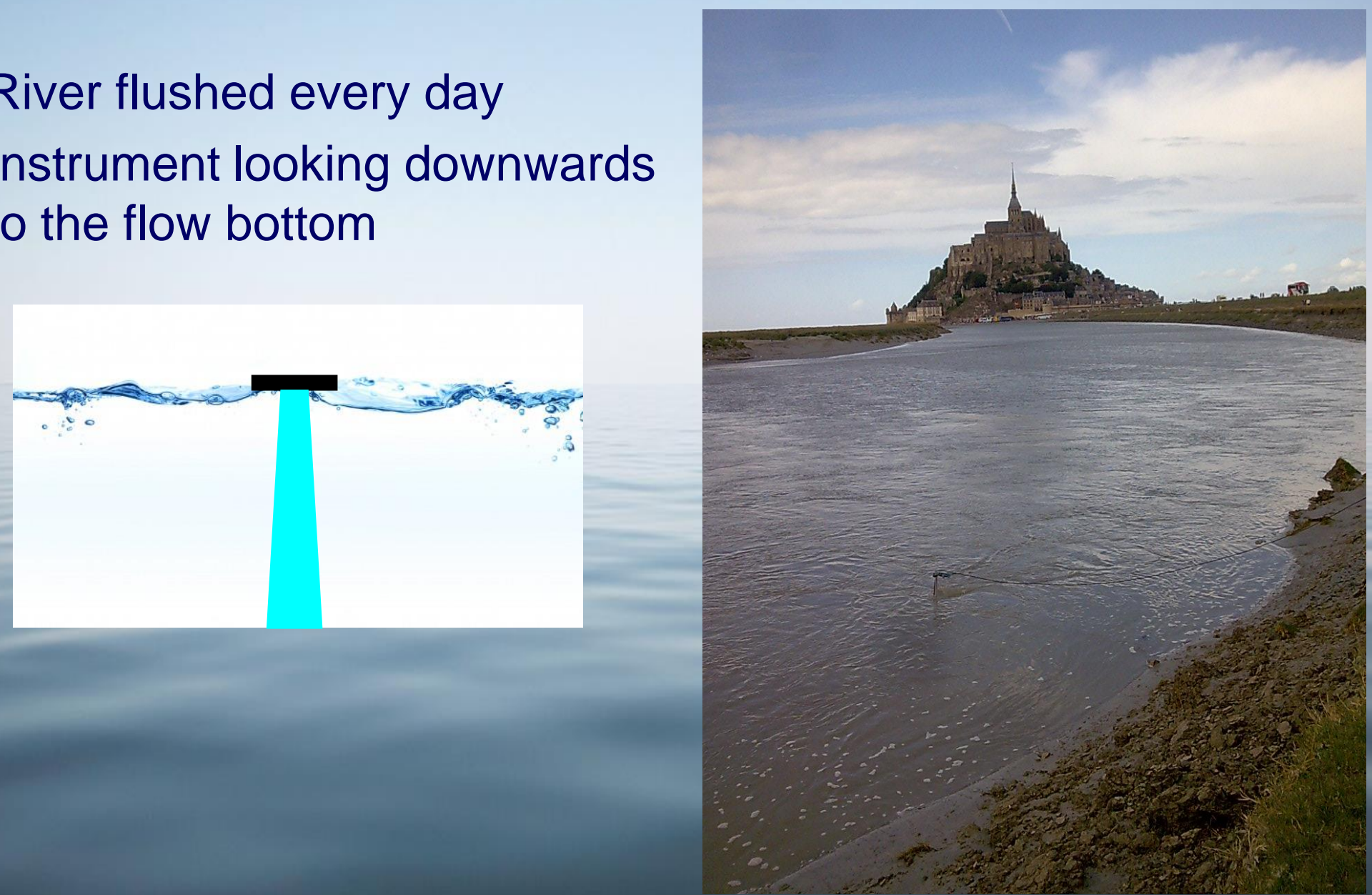

Karlsruher Flussgebietstage 2013, 


\section{Couesnon}

- Amplitude increase with time after the beginning of the flush

- Increase of suspended sediments concentration

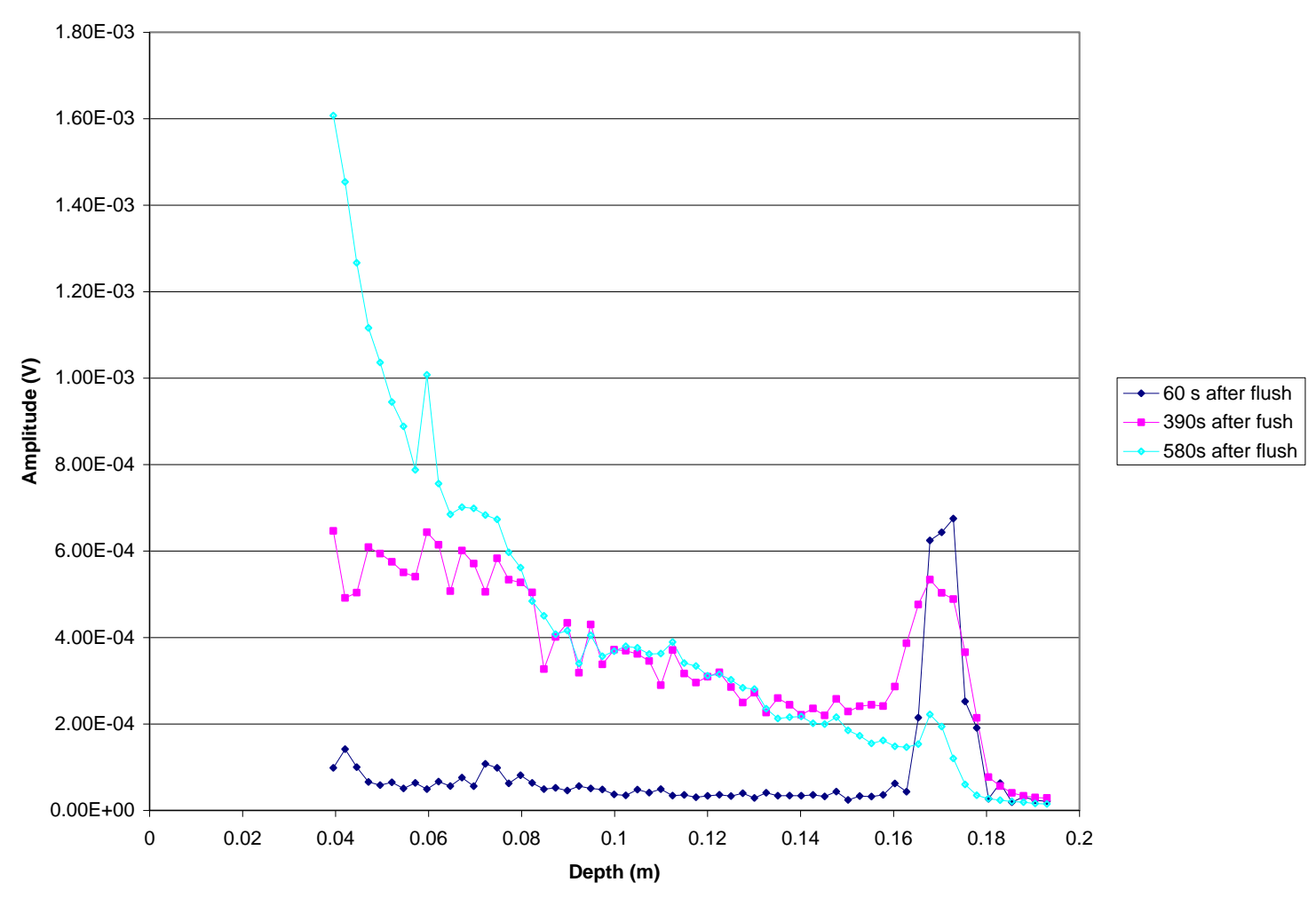




\section{Couesnon}

- Amplitude and velocity evolution with time after the beginning of the flush

- When velocity $>25 \mathrm{~cm} / \mathrm{s}$, amplitude rapidly increases

○ Treshold velocity value for re-suspension?

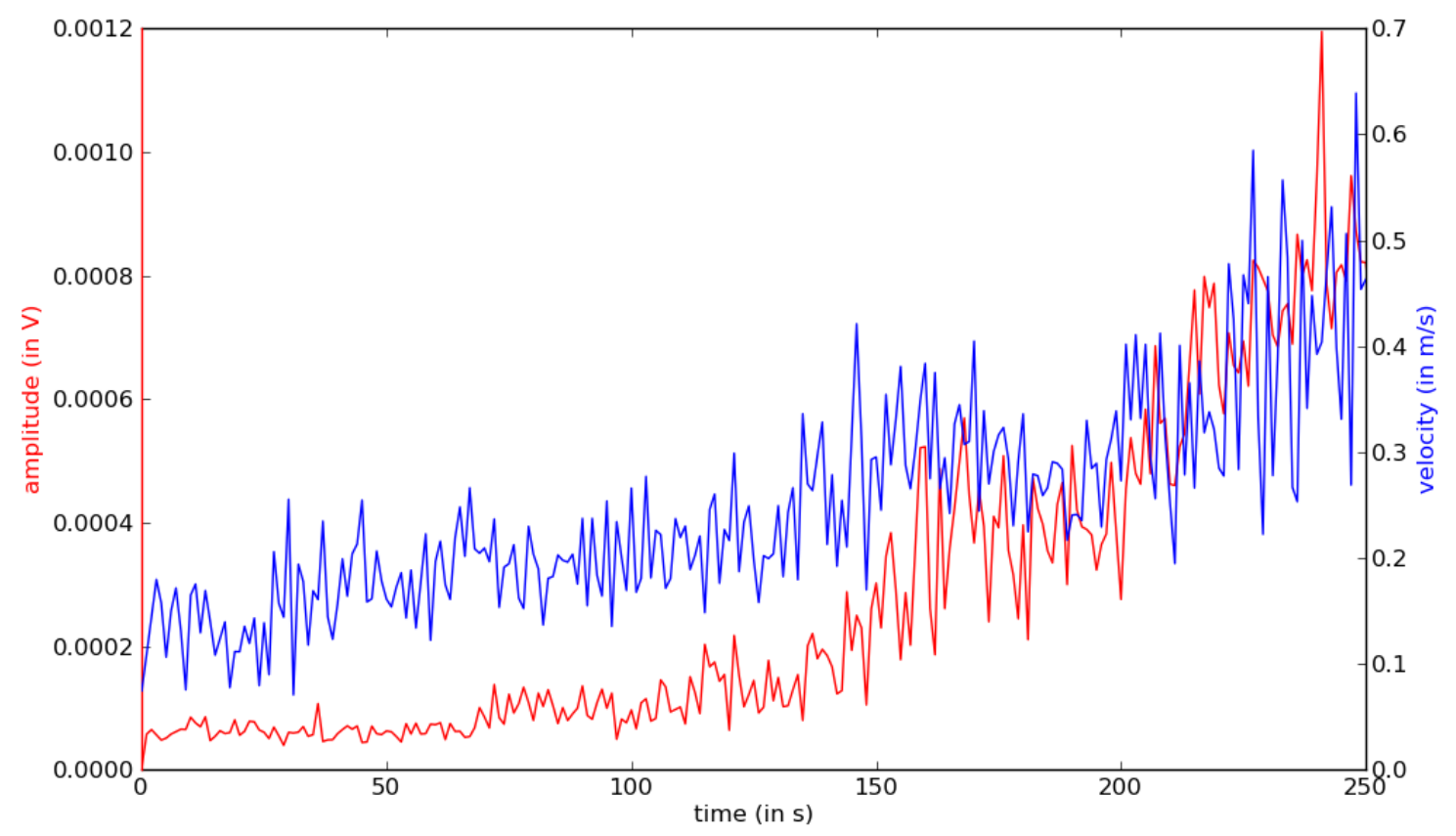




\section{Water treatment inflow}

TiCIJ3E
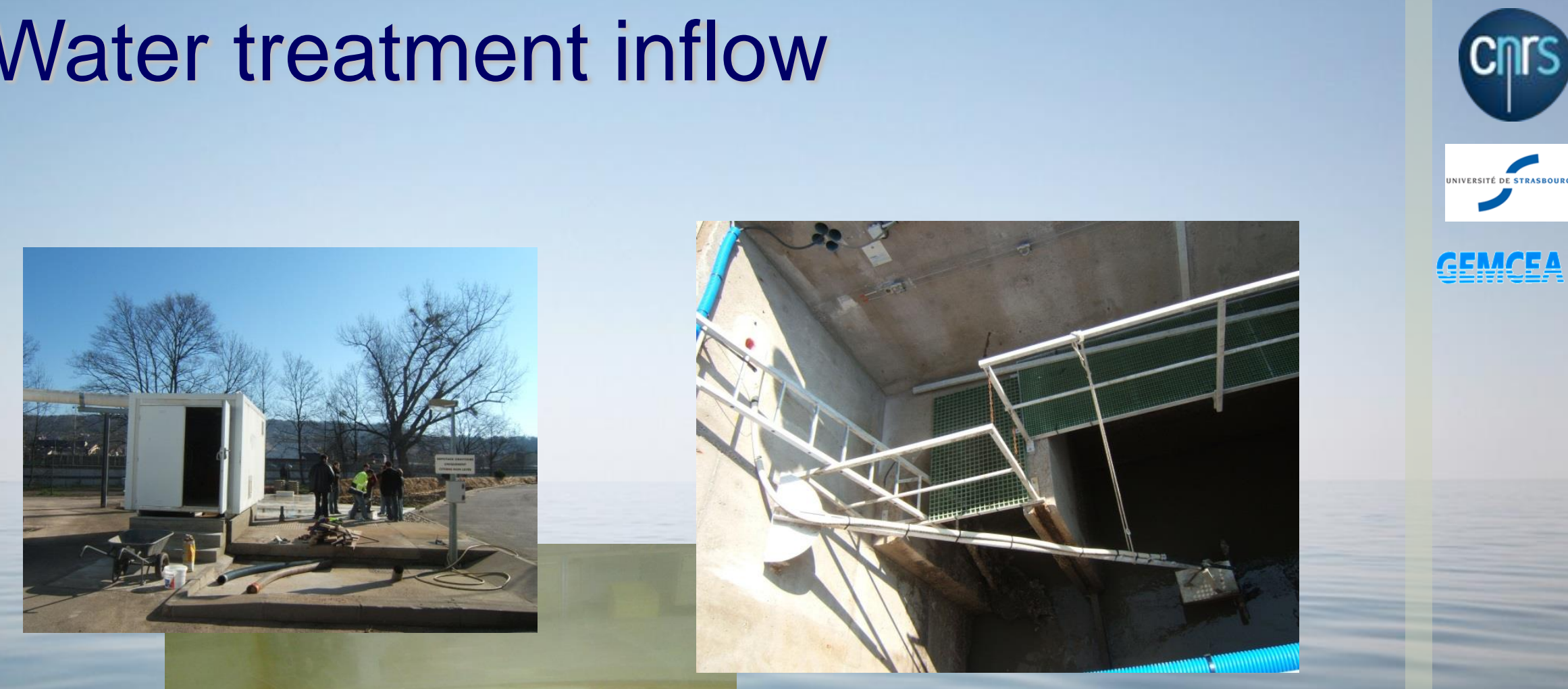

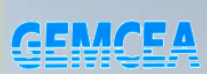

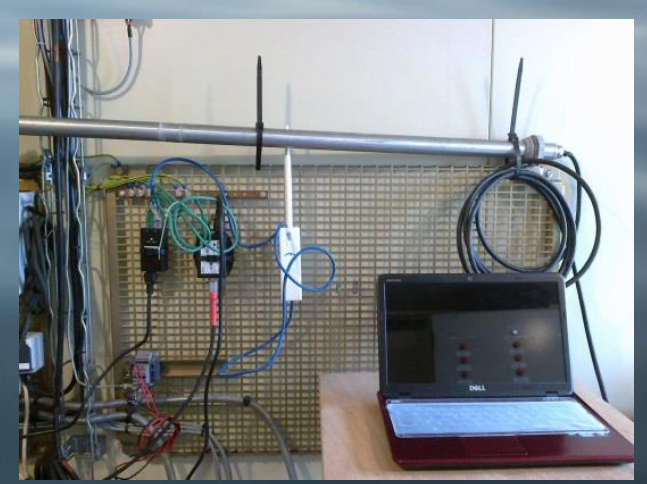

Karlsruher Flussgebietstage 2013,

20-21 June 


\section{Water treatment inflow}

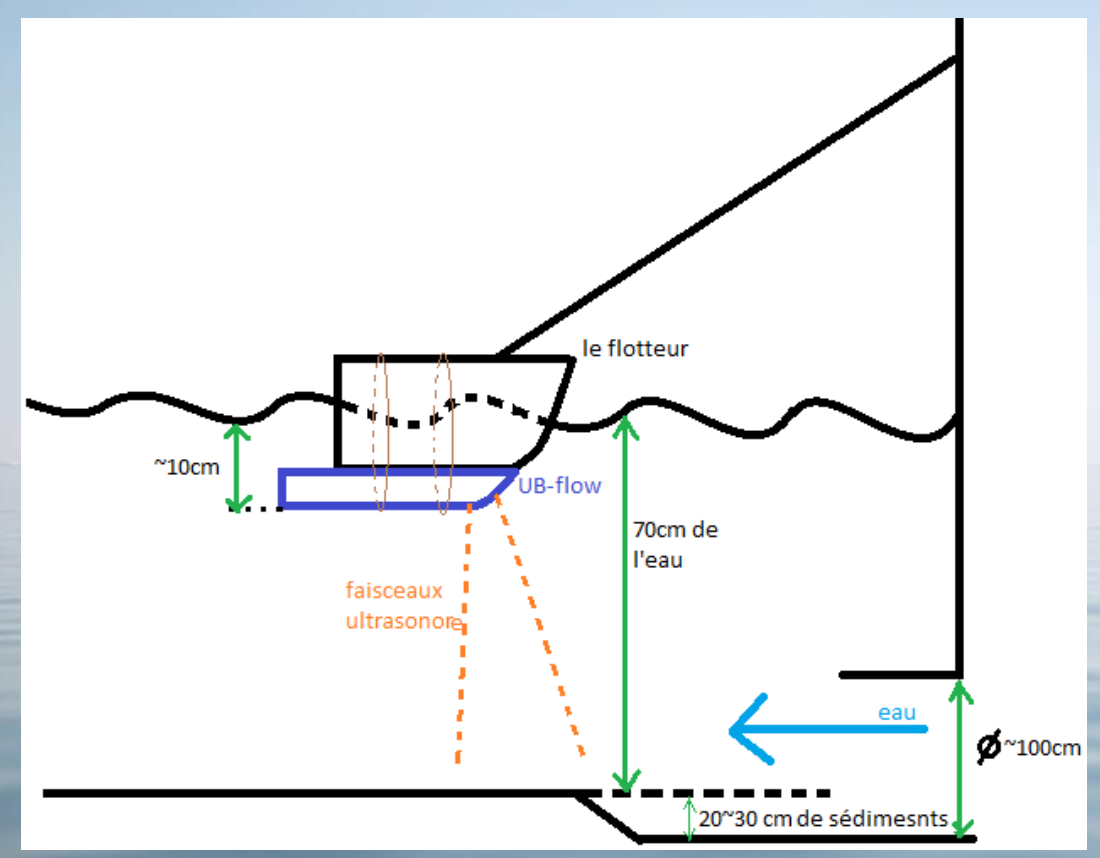

- Instrument looking downwards to the bottom of the flow

- Complex water in- and outlet

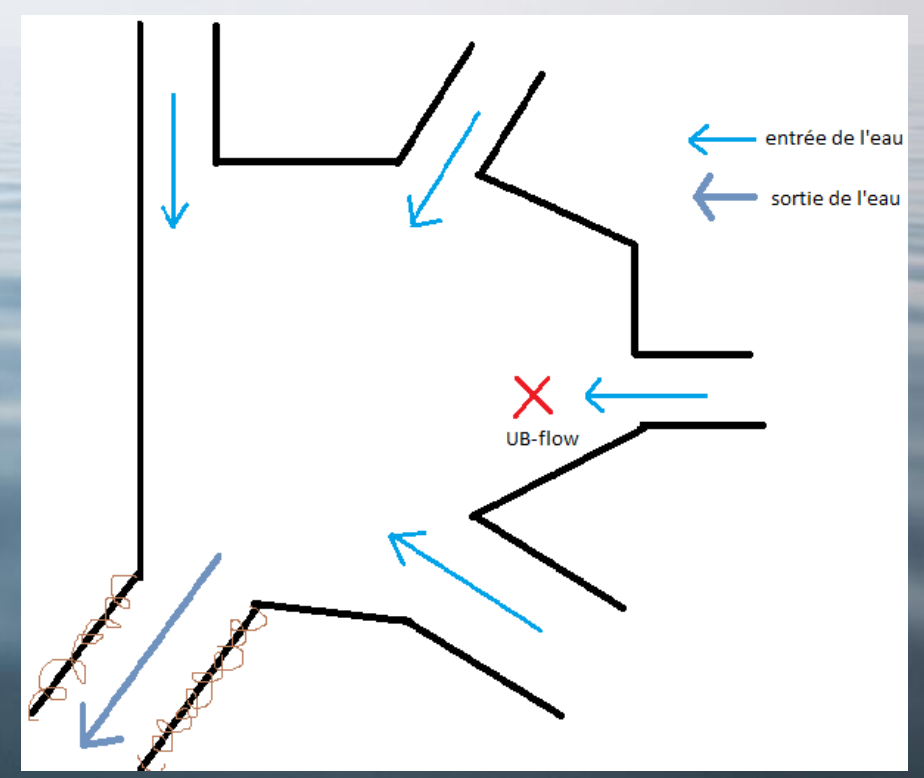




\section{Water treatment inflow}

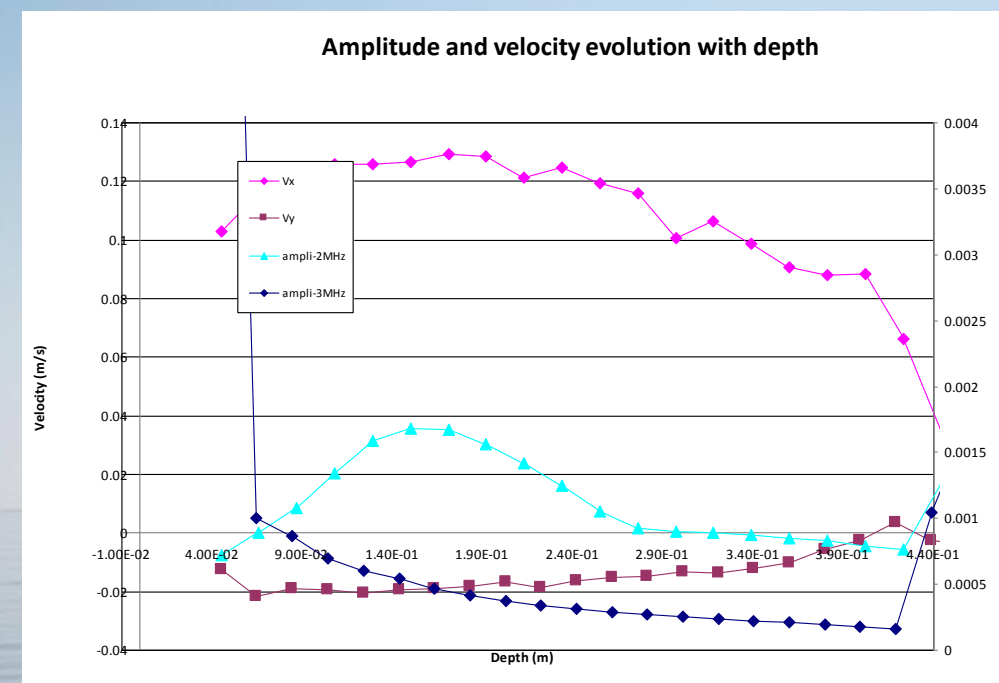

Karlsruher Flussgebietstage 2013, 20-21 June
- Mean values calculated on 8 mn time fractions

- Evolution of velocity and amplitude

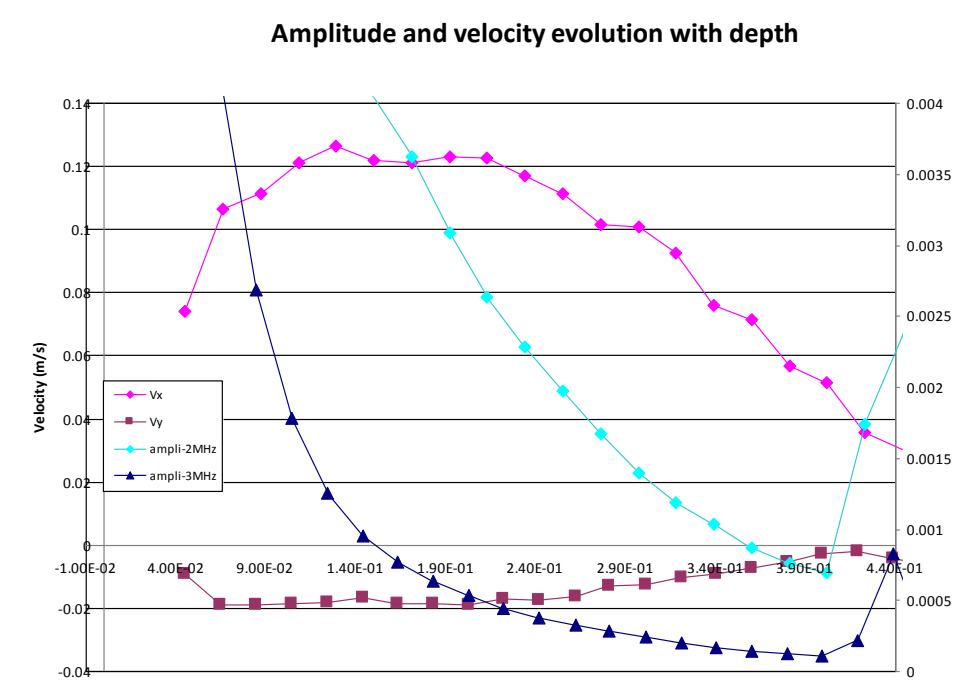

Depth ( $m$ ) 


\section{Water treatment inflow}

- Amplitude evolution for 2 frequencies

- Comparison to TSS concentration obtained by sampling

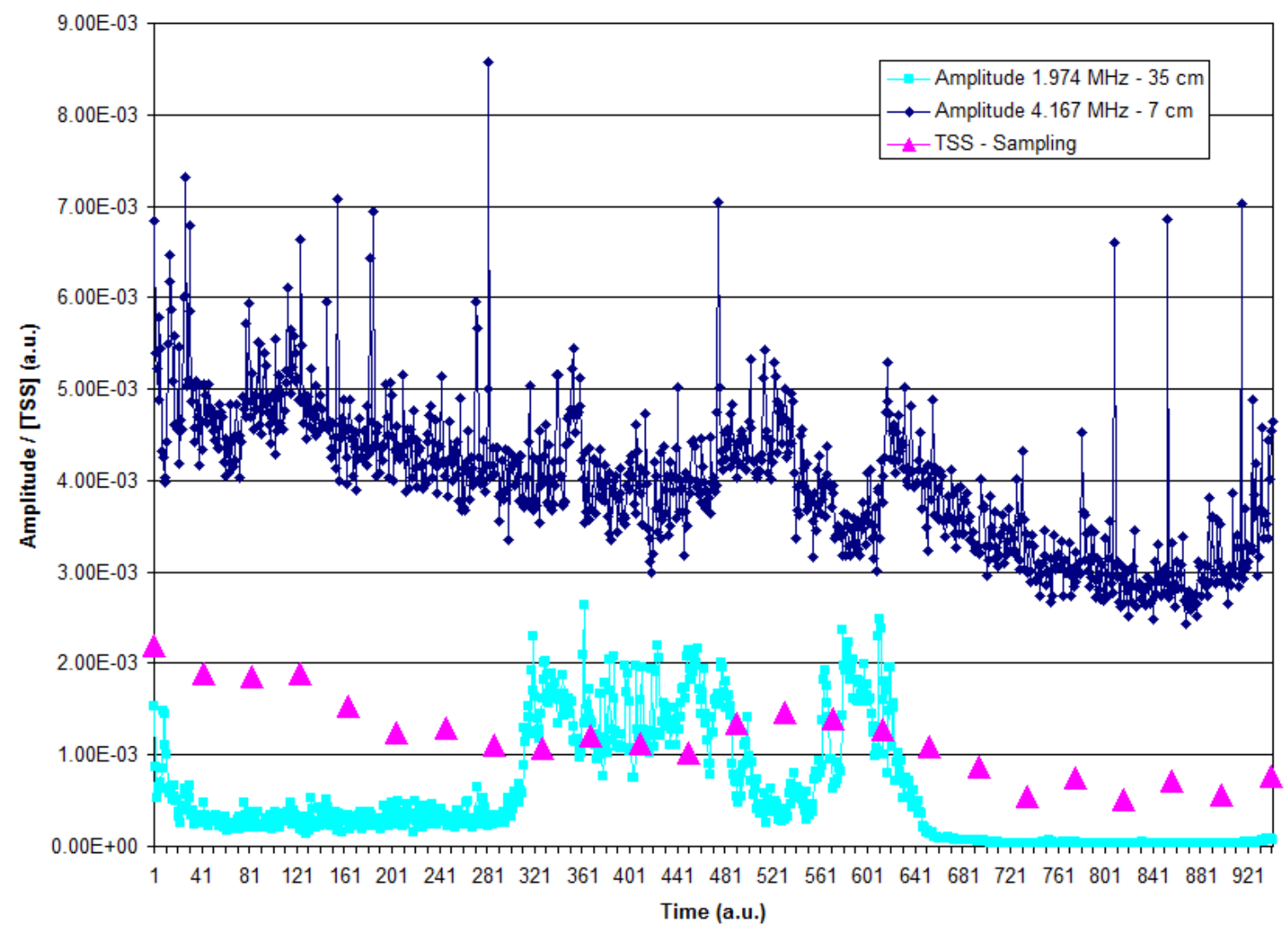




\section{Conclusions}

- Backscattered amplitude representative of acoustic turbidity

- Simple knowledge of acoustic theory allows qualitative data analysis

- Combined analysis of amplitude and velocity can bring more precise information

- => Easy qualitative online monitoring

- => Quantitative analysis would need inversion algorithm 